\title{
Discovery of a lead compound for specific inhibition of type I collagen production in fibrosis
}

\author{
Branko Stefanovic ${ }^{1}$, Heather A. Michaels ${ }^{2}$ and Adel Nefzi ${ }^{*}, 3$
}

${ }^{1}$ Department of Biomedical Sciences, College of Medicine, Florida State University, 1115 West Call Street, Tallahassee, FL, 32306, USA.

${ }^{2}$ Torrey Pines Institute for Molecular Studies, Port Saint Lucie, FL 34987

${ }^{3}$ Florida International University, Port Saint Lucie, FL 34987

\section{Supporting Information}

Page 2: General procedures

Page 3: NMR data

Pages 4-8: Library TPI-2435 distribution

Pages 9-12: Chemical Structures of individual compounds TPI-2659

Pages 13-31: LC-MS of all individual compounds. 


\section{Scaffold library:}

The Scaffold Ranking library composed of 75 different core scaffolds provides a method to triage the available TPI libraries based on activity in a given assay. Each mixture sample (well) contains an equal molar concentration of every structural analog compound of a given scaffold library. In this manner the different core scaffolds available in the TPI collection can be compared based on assay activity. The size of libraries varies between 4,900 to 700,000 compounds.

\section{Positional Scanning Libraries}

Each of the 75 small molecule libraries forming the scaffold ranking library plate is prepared and arranged in positional scanning format. Each of the libraries is made up of 100-200 mixture samples. These mixture samples are systematically formatted so that activities of individual compounds can be predicted from the screening of exponentially fewer samples. The use of positional scanning synthetic combinatorial libraries (PS-SCLs) enables the most active building blocks at each position of a core scaffold to be determined directly from the initial screening data.

General procedure for the synthesis of the individual compounds (TPI-2659): Starting from 100 mg sample of p-methylbenzhydrylamine (MBHA) resin, pre-prepared Boc-L-Pro(4- $\left.\mathrm{N}_{3}\right)-\mathrm{OH}(2 \mathrm{~S}, 4 \mathrm{~S})(3$ eq) was coupled in the presence of diisopropylcarbodiimide (DIC) (3 eq) and hydroxybenzotriazole (HOBt) (3 eq) in anhydrous dimethyl formamide (DMF) for two hours. Completion of the coupling reaction was confirmed by Kaiser test. The azide group was reduced in the presence of tin chloride in anhydrous DMF overnight, and then treated with thiophenol and diisopropyllethylamine (DIEA) in DMF. The generated primary amine was acylated with different commercially available carboxylic acids (10 eq) in the presence if DIC (10 eq) in anhydrous DMF and the completion of the acylation was confirmed by Kaiser test. The Boc group was cleaved in the presence of trifluoroacetic acid (TFA) in dichloromethane (DCM) (55:45) for $30 \mathrm{~min}$ and the amine was neutralized by treating the resin with a $5 \%$ solution of DIEA in DCM (2 times, $5 \mathrm{~min}$ ). The free amine was treated with 4,6-dichloropyrimidine (10 eq) in dioxane at $100^{\circ} \mathrm{C}$ for $24 \mathrm{~h}$. Completion of the reaction was confirmed by LC-MS after cleaving a small amount of the resin. The second chloro group was displaced by treatment of the resin with pierazine (10 eq) in dioxane for $24 \mathrm{~h}$ at $100{ }^{\circ} \mathrm{C}$ and the completion of the reaction was confirmed by LCMS. The free amine of the piperazine ring was acylated with a variety of commercially available carboxylic acids (10 eq) in the presence of DIC. The final desired compound was released from the resin by $\mathrm{HF}$ cleavage at $0{ }^{\circ} \mathrm{C}$ for $1.5 \mathrm{~h}$, and then extracted, lyophilized and purified by preparative HPLC. All the products were confirmed by LC-MS and active compounds with NMR analysis. 
(2S,4S)-1-(6-(4-(3,5-dimethylbenzoyl)piperazin-1-yl)pyrimidin-4-yl)-4-tetradecanamido pyrrolidine-2-carboxamide (2659-17):

${ }^{1} \mathrm{H}-\mathrm{NMR}\left(400 \mathrm{MHz}, \mathrm{D}_{6}\right.$-DMSO, $\left.300 \mathrm{~K}\right): 8.08(\mathrm{~s}, 1 \mathrm{H}), 7.54-7.56(\mathrm{~m}, 1 \mathrm{H}), 7.11(\mathrm{~s}, 1 \mathrm{H}), 7.03(\mathrm{~s}, 2 \mathrm{H}), 5.65$ (bs, $1 \mathrm{H}), 4.32-4.34(\mathrm{~m}, 1 \mathrm{H}), 3.57-3.59(\mathrm{~m}, 4 \mathrm{H}), 3.30-3.40(\mathrm{~m}, 4 \mathrm{H}), 2.31(\mathrm{~s}, 6 \mathrm{H}), 2.03(\mathrm{t}, \mathrm{J}=7.2 \mathrm{~Hz}, 2 \mathrm{H})$, $1.83(\mathrm{~m}, 1 \mathrm{H}), 1.48(\mathrm{~m}, 2 \mathrm{H}), 1.19-1.29(\mathrm{~m}, 18 \mathrm{H}), 0.86(\mathrm{t}, \mathrm{J}=6.48 \mathrm{Kz}, 3 \mathrm{H}) .{ }^{13} \mathrm{C}-\mathrm{NMR}\left(100 \mathrm{MHz}, \mathrm{D}_{6}-\mathrm{DMSO}\right.$, $300 \mathrm{~K}): 172.2,169.9,138.1,136.2,131.3,125.0,118.4,91.5,82.6,59.3,53.1,47.8,36.0,31.7,29.4$, 29.1, 29.0, 25.5, 22.5, 21.2, 14.4. MS (ESI) $\mathrm{m} / \mathrm{z}[\mathrm{M}+\mathrm{H}]^{+}:$634.0. 


\section{Library TPI-2435}

Building blocks used for the synthesis of the library TPI-2435

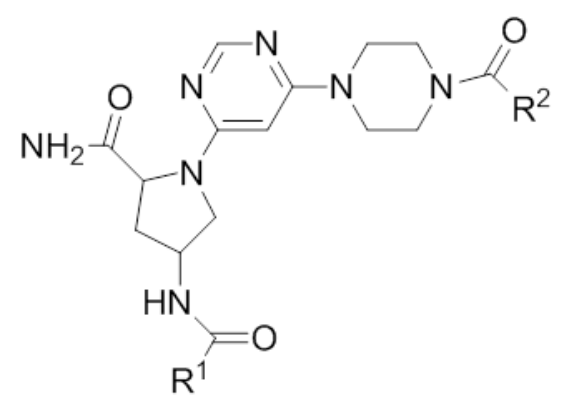

\begin{tabular}{|c|c|c|}
\hline 2435 & & \\
\hline cc & R1 & $\mathbf{R 2}$ \\
\hline Mixtures & Carboxylic Acid Used & Mixtures of carboxylic acids \\
\hline 1 & 1-phenyl-1-cyclopropanecarboxylic acid & $X$ \\
\hline 2 & 2-Phenylbutyric Acid & $\mathrm{X}$ \\
\hline 3 & 3-Phenylbutyric Acid & $\mathrm{X}$ \\
\hline 4 & m-Tolylacetic acid & $\mathrm{X}$ \\
\hline 5 & 3-Fluorophenylacetic Acid & $X$ \\
\hline 6 & 3-Bromophenylacetic Acid & $\mathrm{X}$ \\
\hline 7 & p-Tolyacetic acid & $\mathrm{X}$ \\
\hline 8 & 4-Fluorophenylacetic acid & $\mathrm{X}$ \\
\hline 9 & 3-Methoxyphenylacetic acid & $\mathrm{X}$ \\
\hline 10 & 4-Bromophenylacetic acid & $X$ \\
\hline 11 & 4-Methoxyphenylacetic acid & $\mathrm{X}$ \\
\hline 12 & 3,4-Dimethoxyphenyl acetic acid & $X$ \\
\hline 13 & 4-isobutyl-alpha-Methylphenylacetic Acid & $\mathrm{X}$ \\
\hline 14 & 3,4-Dichlorophenylacetic acid & $\mathrm{X}$ \\
\hline 15 & 3,5-Bis(Trifluoromethyl)-Phenylacetic acid & $\mathrm{X}$ \\
\hline 16 & 3-(3,4-Dimethoxyphenyl)-propionic Acid & $X$ \\
\hline 17 & Phenylacetic acid & $\mathrm{X}$ \\
\hline 18 & 3,4,5-Trimethoxybenzoic acid & $\mathrm{X}$ \\
\hline 19 & Butyric Acid & $\mathrm{X}$ \\
\hline 20 & Heptanoic Acid & $X$ \\
\hline 21 & Isobutyric Acid & $\mathrm{X}$ \\
\hline 22 & 2-Methylbutiric Acid & $X$ \\
\hline 23 & Isovaleric acid & $\mathrm{X}$ \\
\hline 24 & 3-Methylvaleric acid & $\mathrm{X}$ \\
\hline 25 & p-Toluic Acid & $\mathrm{X}$ \\
\hline 26 & cyclopentanecarboxylic acid. & $\mathrm{X}$ \\
\hline 27 & cyclohexanecarboxilic acid & $X$ \\
\hline 28 & cyclohexylacetic acid & $X$ \\
\hline 29 & cyclohexanebutyric acid & $X$ \\
\hline 30 & cycloheptanecarboxylic acid & $\mathrm{X}$ \\
\hline
\end{tabular}




\begin{tabular}{|c|c|c|}
\hline 31 & 1-Adamantaneacetic Acid & $\mathrm{x}$ \\
\hline 32 & cyclobutanecarboxylic acid & $\mathrm{X}$ \\
\hline 33 & 3-cyclopentylpropionic acid & $\mathrm{X}$ \\
\hline 34 & cyclohexanepropionic acid & $\mathrm{x}$ \\
\hline 35 & 4-methyl-1-cyclohexancarboxylic acid & $\mathrm{x}$ \\
\hline 36 & 4-tert-butyl-cyclohexancecarboxylic acid & $\mathrm{x}$ \\
\hline 37 & 4-biphenylacetic acid & $\mathrm{x}$ \\
\hline 38 & 1-Adamantancecarboxylic acid & $\mathrm{x}$ \\
\hline 39 & 4-Methylvaleric acid & $\mathrm{x}$ \\
\hline 40 & 2-norbornaneacetic acid & $\mathrm{x}$ \\
\hline 41 & Hexanoic Acid & $\mathrm{x}$ \\
\hline 42 & Octanoic Acid & $\mathrm{x}$ \\
\hline 43 & 2-Ethylbutyric Acid & $\mathrm{X}$ \\
\hline 44 & Trimethylacetic Acid & $\mathrm{x}$ \\
\hline 45 & Cyclopentylacetic Acid & $\mathrm{x}$ \\
\hline 46 & 3-Cyclopentylpropionic Acid & $\mathrm{x}$ \\
\hline 47 & 2-ethylhexanoic & $\mathrm{x}$ \\
\hline 48 & 2-Phenoxypropionic acid & $\mathrm{x}$ \\
\hline 49 & Benzoic acid & $\mathrm{x}$ \\
\hline 50 & 2-Chlorobenzoic acid & $\mathrm{x}$ \\
\hline 51 & 2-(P-Toluoyl)- Benzoic acid & $\mathrm{x}$ \\
\hline 52 & m-Toluic acid & $\mathrm{x}$ \\
\hline 53 & 4-Fluorobenzoic Acid & $\mathrm{x}$ \\
\hline 54 & 4-Bromobenzoic Acid & $\mathrm{x}$ \\
\hline 55 & 4-Ethylbiphenyl-4'-carboxylic acid & $\mathrm{x}$ \\
\hline 56 & 3,4-Dimethylbenzoic acid & $\mathrm{x}$ \\
\hline 57 & 4-Biphenylcarboxylic Acid & $\mathrm{x}$ \\
\hline 58 & 2-BenzoylBenzoic acid & $\mathrm{x}$ \\
\hline 59 & 1-Naphthoic acid & $\mathrm{x}$ \\
\hline 60 & 2-Furoic acid & $\mathrm{x}$ \\
\hline 61 & Indole-3-acetic acid & $\mathrm{x}$ \\
\hline 62 & Tert-butyl acetic acid & $\mathrm{x}$ \\
\hline 63 & 3,3-diphenylpropionic acid & $\mathrm{x}$ \\
\hline 64 & 5-Methyl-2-pyrazinecarboxylic acid & $\mathrm{x}$ \\
\hline 65 & 2-Benzimidazolepropionic acid & $\mathrm{X}$ \\
\hline 66 & 4-Phenylbutyric acid & $\mathrm{x}$ \\
\hline 67 & 5-Bromo-2-furoic acid & $\mathrm{X}$ \\
\hline 68 & 3-Bromopropionic acid & $\mathrm{x}$ \\
\hline 69 & 3,3,3-triphenylpropionic acid & $\mathrm{x}$ \\
\hline 70 & Myristic Acid & $\mathrm{x}$ \\
\hline 71 & 2-chloropyrimidine & $\mathrm{X}$ \\
\hline 72 & \multirow{5}{*}{$\begin{array}{l}\text { 2-Naphthoxyacetic acid } \\
\text { 2-Phenyl-4-quinolinecarboxylic acid } \\
\text { 3-(3,4,5-trimethoxyphenyl)-Propionic acid } \\
\text { 3,4-(methylenedioxy)-phenylacetic acid } \\
\text { 1-Methyl-2-pyrrolecarboxylic acid }\end{array}$} & $\mathrm{X}$ \\
\hline 73 & & $\mathrm{X}$ \\
\hline 74 & & $\mathrm{X}$ \\
\hline 75 & & $\mathrm{x}$ \\
\hline 76 & & $\mathrm{x}$ \\
\hline
\end{tabular}




\begin{tabular}{|c|c|c|}
\hline 77 & \multirow{2}{*}{$\begin{array}{l}\text { 1-phenyl-1-cyclopentane carboxylic acid } \\
\text { 2,3,4,5,6-pentafluorophenylacetic acid }\end{array}$} & $\mathrm{X}$ \\
\hline 78 & & $\mathrm{X}$ \\
\hline 79 & 3,5-Bis(Trifluoromethyl)-benzoic acid & $\mathrm{X}$ \\
\hline 80 & \multirow{4}{*}{$\begin{array}{l}\text { 4,5-dibromo-1H-pyrrole-2-carboxylic acid } \\
\text { 4-isopropylbenzoic acid } \\
\text { 5-methyl-3-phenylisoxazole-4-carboxylic } \\
\text { acid } \\
\text { 2-methyl-4-nitro-1-imidazole-propionic acid }\end{array}$} & $\mathrm{X}$ \\
\hline 81 & & $\mathrm{X}$ \\
\hline 82 & & $X$ \\
\hline 83 & & $\mathrm{X}$ \\
\hline 84 & 2-Methylcyclopropanecarboxylic Acid & $X$ \\
\hline 85 & 3,3-Dimethylacrylic Acid & $\mathrm{X}$ \\
\hline 86 & Dicyclohexylacetic Acid & $\mathrm{X}$ \\
\hline 87 & Tert-Butylacetic Acid & $\mathrm{X}$ \\
\hline 88 & Trans-3-Hexenoic Acid & $\mathrm{X}$ \\
\hline 89 & 2-Napthylacetic acid & $\mathrm{X}$ \\
\hline 90 & 3,4,5-Triethoxybenzoic acid & $\mathrm{X}$ \\
\hline 91 & Diphenylacetic acid & $\mathrm{X}$ \\
\hline 92 & 2-pyrazine carboxylic acid & $\mathrm{X}$ \\
\hline 93 & (Phenylthio) Acetic Acid & $X$ \\
\hline 94 & 2,4-Dichlorobenzoic acid & $\mathrm{X}$ \\
\hline 95 & 2,4-DimethoxyBenzoic acid & $\mathrm{X}$ \\
\hline 96 & 3,4-Dimethoxybenzoic acid & $\mathrm{X}$ \\
\hline 97 & 3,5-Dimethylbenzoic acid & $\mathrm{X}$ \\
\hline 98 & 3-Benzoylpropionic acid & $\mathrm{X}$ \\
\hline 99 & 2-Cyclopentene-1-Acetic Acid & $X$ \\
\hline 100 & 4-Methylcyclohexaneacetic Acid & $X$ \\
\hline \multirow[t]{2}{*}{101} & 4-tert-Butyl-Cyclohexanecarboxylic Acid & $\mathrm{X}$ \\
\hline & Mixtures of carboxylic acids & Carboxylic Acid \\
\hline 102 & $\mathrm{x}$ & 1-phenyl-1-cyclopropanecarboxylic acid \\
\hline 103 & $x$ & 2-Phenylbutyric Acid \\
\hline 104 & $x$ & 3-Phenylbutyric Acid \\
\hline 105 & $x$ & m-Tolylacetic acid \\
\hline 106 & $x$ & 3-Fluorophenylacetic Acid \\
\hline 107 & $x$ & 3-Bromophenylacetic Acid \\
\hline 108 & $\mathrm{x}$ & p-Tolyacetic acid \\
\hline 109 & $x$ & 4-Fluorophenylacetic acid \\
\hline 110 & $x$ & 3-Methoxyphenylacetic acid \\
\hline 111 & $x$ & 4-Bromophenylacetic acid \\
\hline 112 & $x$ & 4-Methoxyphenylacetic acid \\
\hline 113 & $x$ & 3,4-Dimethoxyphenyl acetic acid \\
\hline 114 & $x$ & 4-isobutyl-alpha-Methylphenylacetic Acid \\
\hline 115 & $x$ & 3,4-Dichlorophenylacetic acid \\
\hline 116 & $x$ & 3,5-Bis(Trifluoromethyl)-Phenylacetic acid \\
\hline 117 & $x$ & 3-(3,4-Dimethoxyphenyl)-propionic Acid \\
\hline 118 & $x$ & Phenylacetic acid \\
\hline 119 & $x$ & 3,4,5-Trimethoxybenzoic acid \\
\hline
\end{tabular}




\begin{tabular}{|c|c|c|}
\hline 120 & $\mathrm{x}$ & Butyric Acid \\
\hline 121 & $\mathrm{x}$ & Heptanoic Acid \\
\hline 122 & $\mathrm{x}$ & Isobutyric Acid \\
\hline 123 & $\mathrm{x}$ & 2-Methylbutiric Acid \\
\hline 124 & $\mathrm{x}$ & Isovaleric acid \\
\hline 125 & $\mathrm{x}$ & 3-Methylvaleric acid \\
\hline 126 & $x$ & p-Toluic Acid \\
\hline 127 & $\mathrm{x}$ & cyclopentanecarboxylic acid. \\
\hline 128 & $\mathrm{x}$ & cyclohexanecarboxilic acid \\
\hline 129 & $\mathrm{x}$ & cyclohexylacetic acid \\
\hline 130 & $\mathrm{x}$ & cyclohexanebutyric acid \\
\hline 131 & $\mathrm{x}$ & cycloheptanecarboxylic acid \\
\hline 132 & $\mathrm{x}$ & 1-Adamantaneacetic Acid \\
\hline 133 & $x$ & cyclobutanecarboxylic acid \\
\hline 134 & $\mathrm{x}$ & 3-cyclopentylpropionic acid \\
\hline 135 & $\mathrm{x}$ & cyclohexanepropionic acid \\
\hline 136 & $\mathrm{x}$ & 4-methyl-1-cyclohexancarboxylic acid \\
\hline 137 & $x$ & 4-tert-butyl-cyclohexancecarboxylic acid \\
\hline 138 & $\mathrm{x}$ & 4-biphenylacetic acid \\
\hline 139 & $x$ & 1-Adamantancecarboxylic acid \\
\hline 140 & $\mathrm{x}$ & 4-Methylvaleric acid \\
\hline 141 & $\mathrm{x}$ & 2-norbornaneacetic acid \\
\hline 142 & $x$ & Hexanoic Acid \\
\hline 143 & $x$ & Octanoic Acid \\
\hline 144 & $\mathrm{x}$ & 2-Ethylbutyric Acid \\
\hline 145 & $\mathrm{x}$ & Trimethylacetic Acid \\
\hline 146 & $\mathrm{x}$ & Cyclopentylacetic Acid \\
\hline 147 & $\mathrm{x}$ & 3-Cyclopentylpropionic Acid \\
\hline 148 & $\mathrm{x}$ & 2-ethylhexanoic \\
\hline 149 & $\mathrm{x}$ & 2-Phenoxypropionic acid \\
\hline 150 & $x$ & Benzoic acid \\
\hline 151 & $\mathrm{x}$ & 2-Chlorobenzoic acid \\
\hline 152 & $\mathrm{x}$ & 2-(P-Toluoyl)- Benzoic acid \\
\hline 153 & $x$ & m-Toluic acid \\
\hline 154 & $\mathrm{x}$ & 4-Fluorobenzoic Acid \\
\hline 155 & $x$ & 4-Bromobenzoic Acid \\
\hline 156 & $x$ & 4-Ethylbiphenyl-4'-carboxylic acid \\
\hline 157 & $x$ & 3,4-Dimethylbenzoic acid \\
\hline 158 & $\mathrm{x}$ & 4-Biphenylcarboxylic Acid \\
\hline 159 & $x$ & 2-BenzoylBenzoic acid \\
\hline 160 & $x$ & 1-Naphthoic acid \\
\hline 161 & $x$ & 2-Furoic acid \\
\hline 162 & $\mathrm{x}$ & Indole-3-acetic acid \\
\hline 163 & $x$ & Tert-butyl acetic acid \\
\hline 164 & $\mathrm{x}$ & 3,3-diphenylpropionic acid \\
\hline 165 & $x$ & 5-Methyl-2-pyrazinecarboxylic acid \\
\hline
\end{tabular}




\begin{tabular}{|c|c|c|}
\hline 166 & $\mathrm{x}$ & 2-Benzimidazolepropionic acid \\
\hline 167 & $\mathrm{x}$ & 4-Phenylbutyric acid \\
\hline 168 & $\mathrm{x}$ & 5-Bromo-2-furoic acid \\
\hline 169 & $\mathrm{x}$ & 3-Bromopropionic acid \\
\hline 170 & $\mathrm{x}$ & 3,3,3-triphenylpropionic acid \\
\hline 171 & $\mathrm{x}$ & Myristic Acid \\
\hline 172 & $\mathrm{x}$ & 2-chloropyrimidine \\
\hline 173 & $x$ & \multirow{7}{*}{$\begin{array}{c}\text { 2-Naphthoxyacetic acid } \\
\text { 2-Phenyl-4-quinolinecarboxylic acid } \\
\text { 3-(3,4,5-trimethoxyphenyl)-Propionic acid } \\
\text { 3,4-(methylenedioxy)-phenylacetic acid } \\
\text { 1-Methyl-2-pyrrolecarboxylic acid } \\
\text { 1-phenyl-1-cyclopentane carboxylic acid } \\
\text { 2,3,4,5,6-pentafluorophenylacetic acid }\end{array}$} \\
\hline 174 & $\mathrm{x}$ & \\
\hline 175 & $x$ & \\
\hline 176 & $\mathrm{x}$ & \\
\hline 177 & $\mathrm{x}$ & \\
\hline 178 & $x$ & \\
\hline 179 & $x$ & \\
\hline 180 & $\mathrm{x}$ & 3,5-Bis(Trifluoromethyl)-benzoic acid \\
\hline 181 & $\mathrm{x}$ & \multirow{4}{*}{$\begin{array}{l}\text { 4,5-dibromo-1H-pyrrole-2-carboxylic acid } \\
\text { 4-isopropylbenzoic acid } \\
\text { 5-methyl-3-phenylisoxazole-4-carboxylic acio } \\
\text { 2-methyl-4-nitro-1-imidazole-propionic acid }\end{array}$} \\
\hline 182 & $x$ & \\
\hline 183 & $\mathrm{x}$ & \\
\hline 184 & $\mathrm{x}$ & \\
\hline 185 & $\mathrm{x}$ & 2-Methylcyclopropanecarboxylic Acid \\
\hline 186 & $\mathrm{x}$ & 3,3-Dimethylacrylic Acid \\
\hline 187 & $\mathrm{x}$ & Dicyclohexylacetic Acid \\
\hline 188 & $\mathrm{x}$ & Tert-Butylacetic Acid \\
\hline 189 & $x$ & Trans-3-Hexenoic Acid \\
\hline 190 & $x$ & 2-Napthylacetic acid \\
\hline 191 & $x$ & 3,4,5-Triethoxybenzoic acid \\
\hline 192 & $\mathrm{x}$ & Diphenylacetic acid \\
\hline 193 & $x$ & 2-pyrazine carboxylic acid \\
\hline 194 & $\mathrm{x}$ & (Phenylthio) Acetic Acid \\
\hline 195 & $\mathrm{x}$ & 2,4-Dichlorobenzoic acid \\
\hline 196 & $x$ & 2,4-DimethoxyBenzoic acid \\
\hline 197 & $x$ & 3,4-Dimethoxybenzoic acid \\
\hline 198 & $\mathrm{x}$ & 3,5-Dimethylbenzoic acid \\
\hline 199 & $x$ & 3-Benzoylpropionic acid \\
\hline 200 & $x$ & 2-Cyclopentene-1-Acetic Acid \\
\hline 201 & $x$ & 4-Methylcyclohexaneacetic Acid \\
\hline 202 & $x$ & 4-tert-Butyl-Cyclohexanecarboxylic Acid \\
\hline 203 & & \\
\hline
\end{tabular}


<smiles>CCC(C)CC(=O)NC1CC(C(N)=O)N(c2cc(N3CCN(C(=O)Cc4ccc(Cl)c(Cl)c4)CC3)ncn2)C1</smiles><smiles>C/C=C(/CCl)C(=O)NC1CC(C(N)=O)N(c2cc(N3CCN(C(=O)Cc4ccc(C)c(Cl)c4)CC3)ncn2)C1</smiles>

$2659-3$

Exact Mass: 649.09

Molecular Weight: 651.37

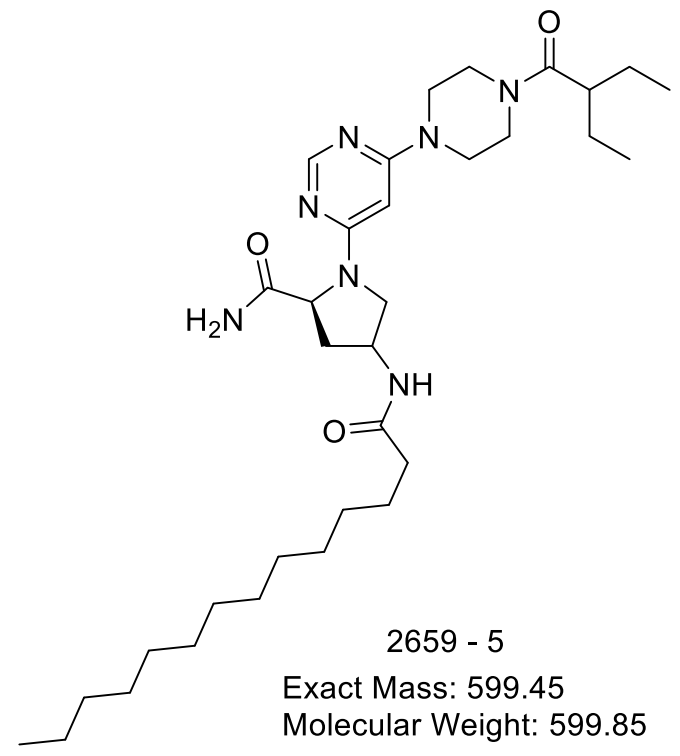<smiles>CCC(C)CC(=O)NC1CC(C(N)=O)N(c2cc(N3CCN(C(=O)C(CC)CC)CC3)ncn2)C1</smiles>

Exact Mass: 487.33 Molecular Weight: 487.64<smiles>CCC(CC)C(=O)N1CCN(c2cc(N3CC(NC(=O)c4ccc(Cl)cc4Cl)C[C@H]3C(N)=O)ncn2)CC1</smiles>

Exact Mass: 561.20

Molecular Weight: 562.49 


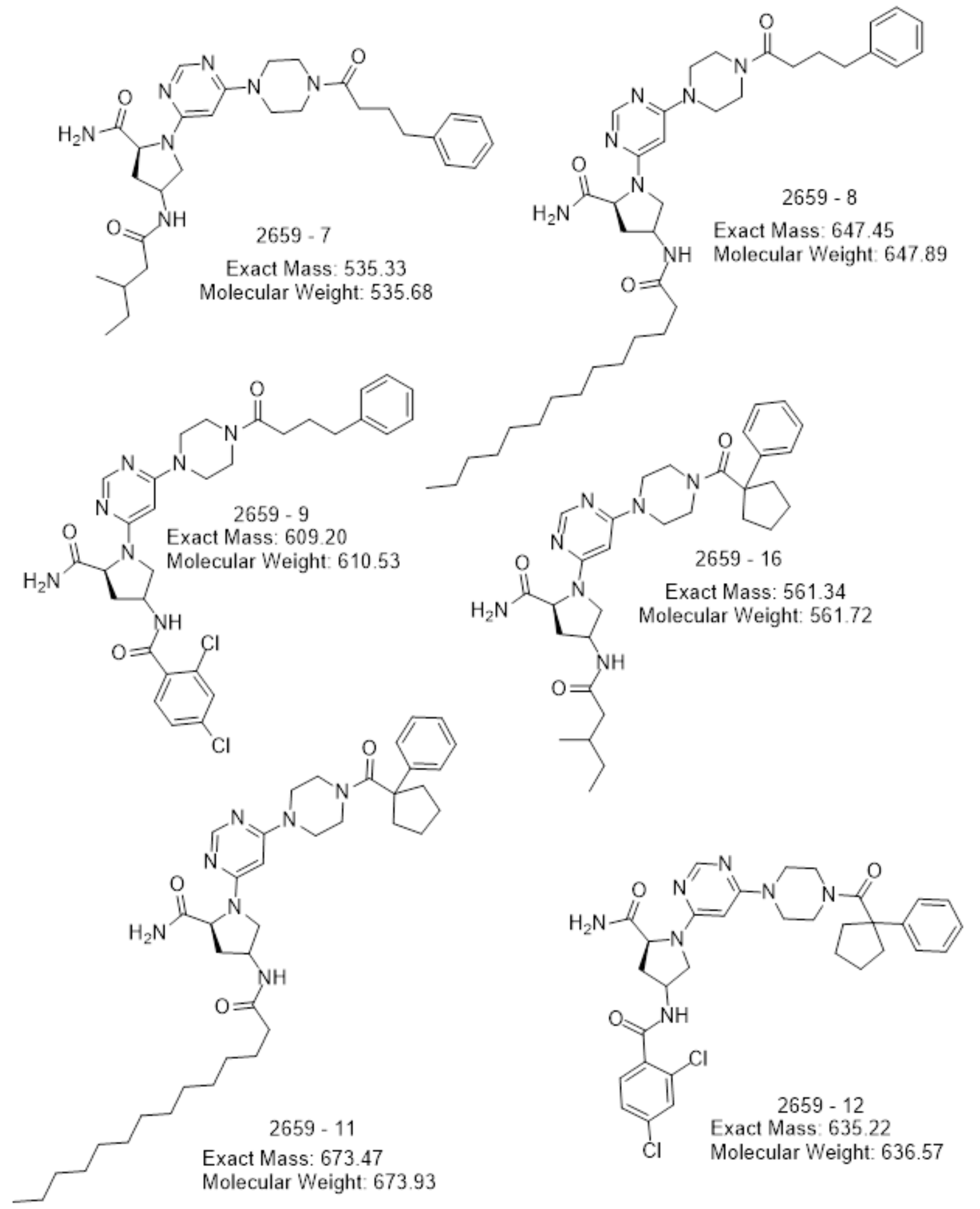


<smiles>CCC(C)CC(=O)NC1C[C@H](C(N)=O)N(c2cc(N3CCN(C(=O)c4ccc(Cl)cc4Cl)CC3)ncn2)C1</smiles>

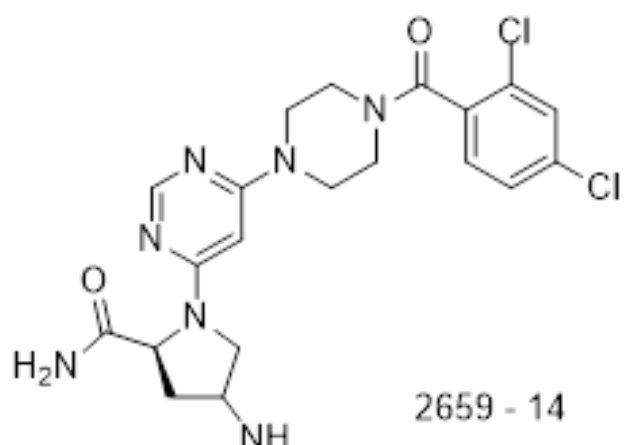

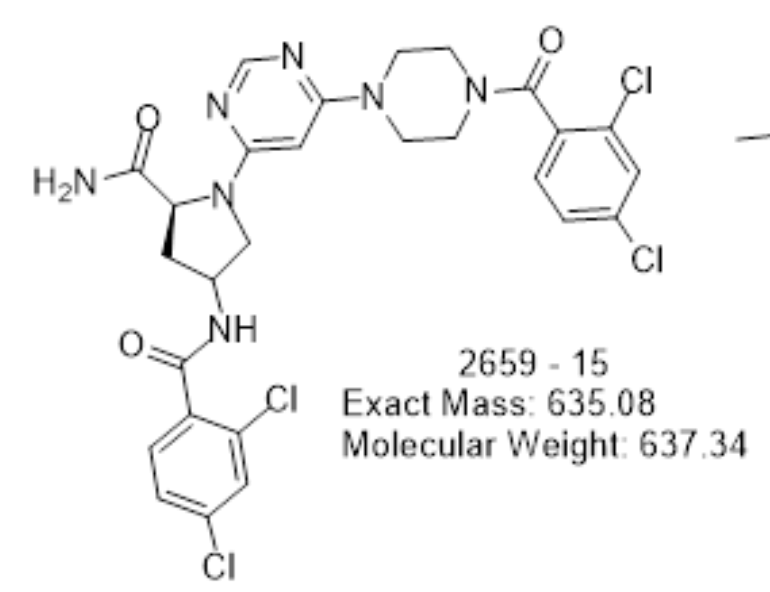<smiles>CCC(C)CC(=O)NC1CC(C(N)=O)N(c2cc(N3CCN(C(=O)c4cc(C)cc(C)c4)CC3)ncn2)C1</smiles><smiles>CCCCCCCCCC(=O)NC1CC(C(N)=O)N(c2cc(N3CCN(C(=O)c4cc(C)cc(C)c4)CC3)ncn2)C1</smiles><smiles>Cc1cc(C)cc(C(=O)N2CCN(c3cc(N4CC(NC(=O)c5ccc(Cl)cc5Cl)C[C@H]4C(N)=O)ncn3)CC2)c1</smiles> 

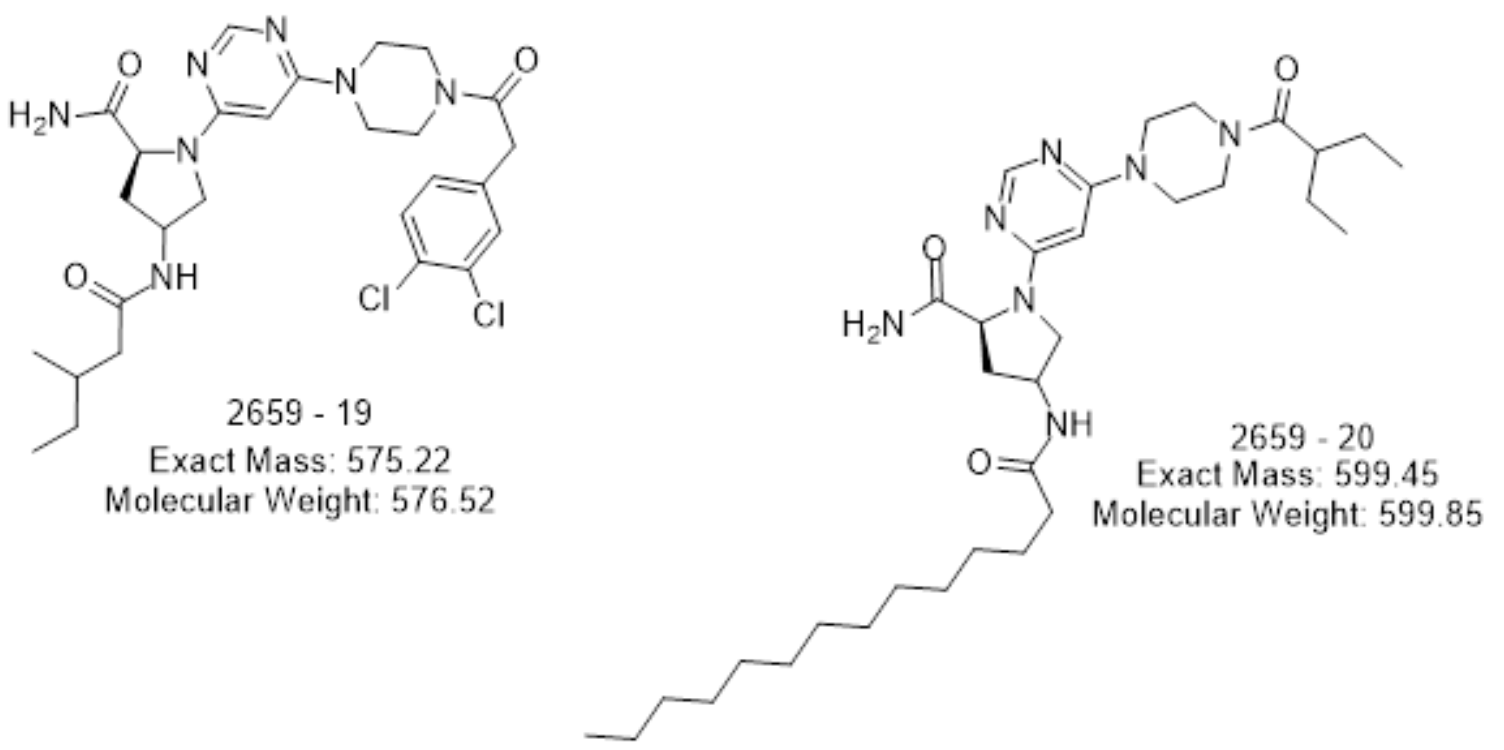


\section{LCMS of all individual compounds}

2659-1

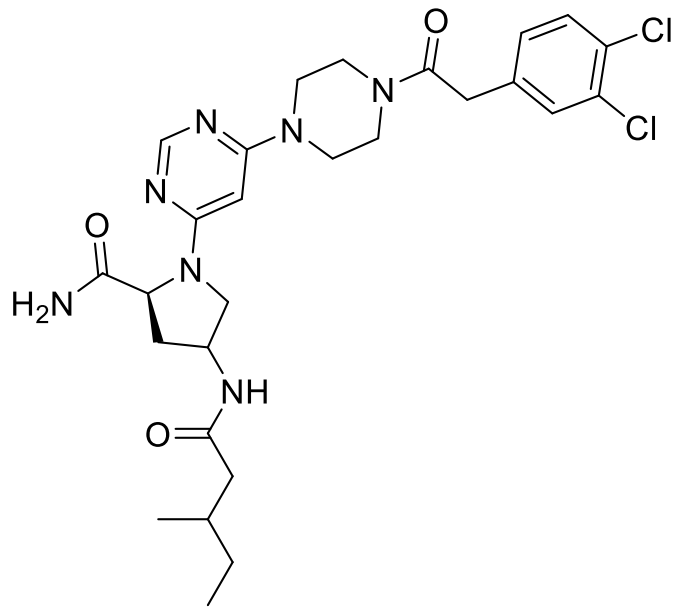

Chemical Formula: $\mathrm{C}_{27} \mathrm{H}_{35} \mathrm{Cl}_{2} \mathrm{~N}_{7} \mathrm{O}_{3}$

Exact Mass: 575.22

Molecular Weight: 576.52

uAU

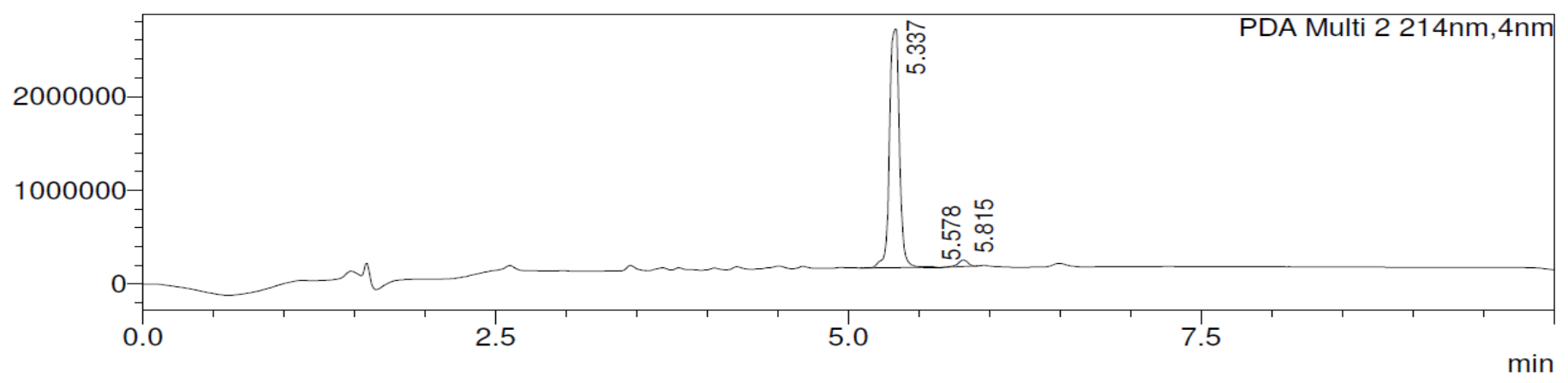

Peak\#:4 R.Time:5.369(Scan\#:1289)

MassPeaks:34

Spectrum Mode:Averaged 5.363-5.371(1288-1290)

BG Mode:Calc Segment 1 - Event 1

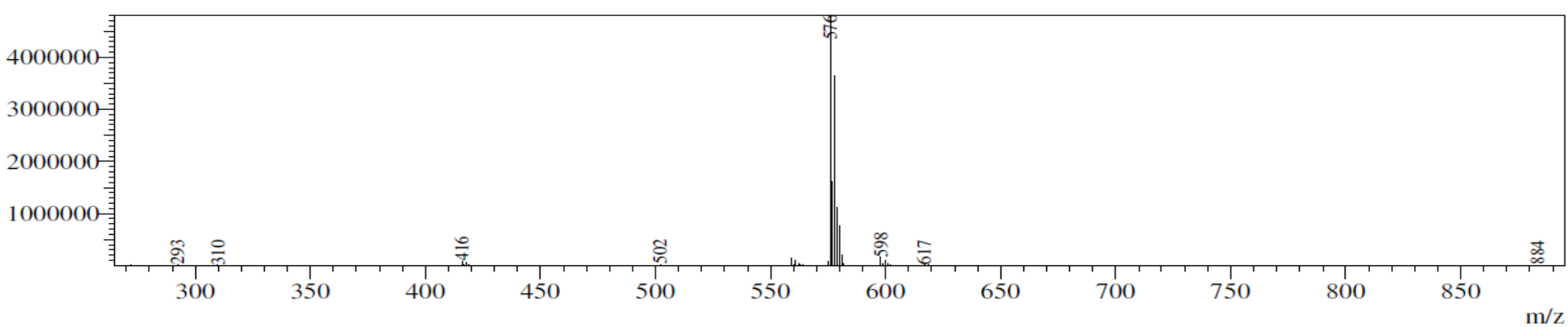



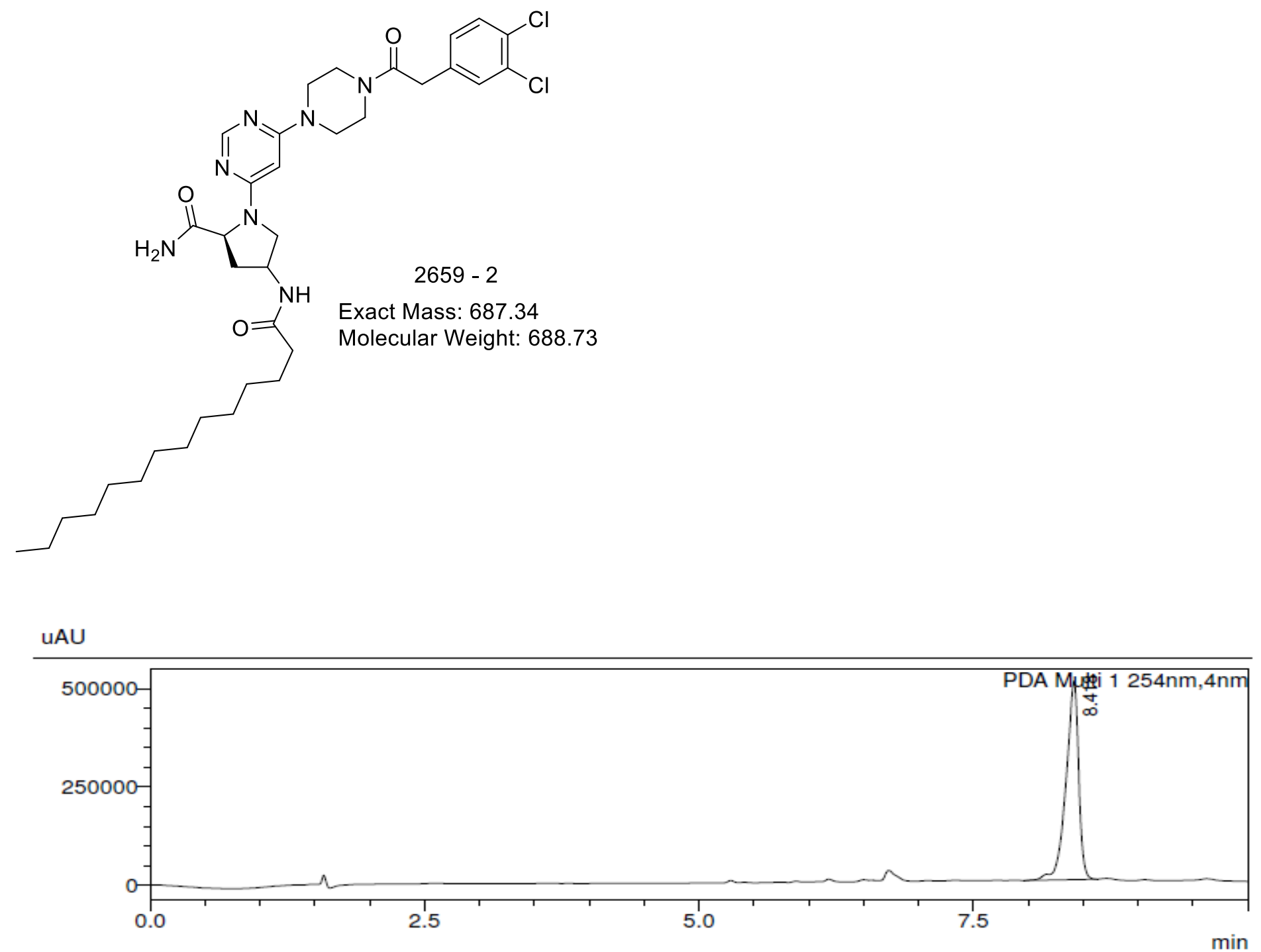

Peak\#:5 R. Time:8.461(Scan\#: 2032)

MassPeaks:28

Spectrum Mode:Averaged 8.458-8.467(2031-2033)

BG Mode:Calc Segment 1 - Event 1

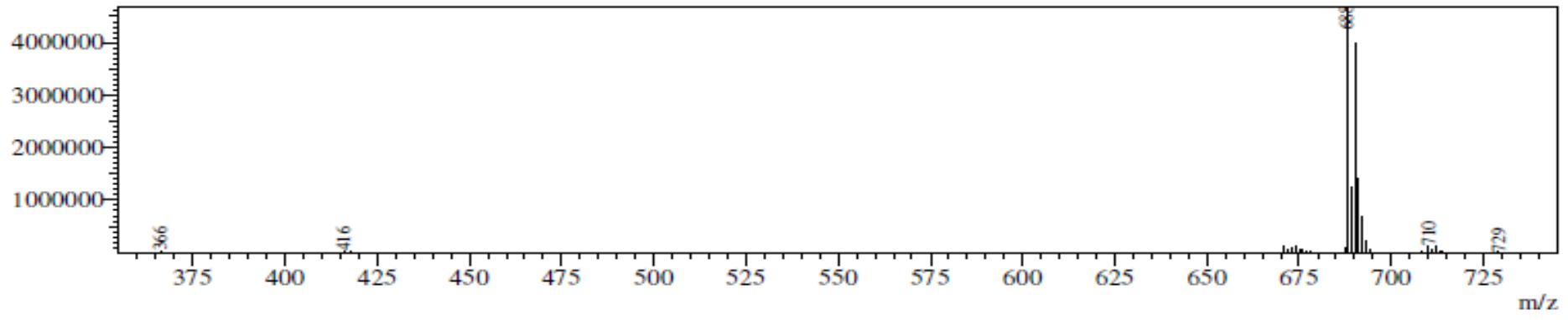


2659-3

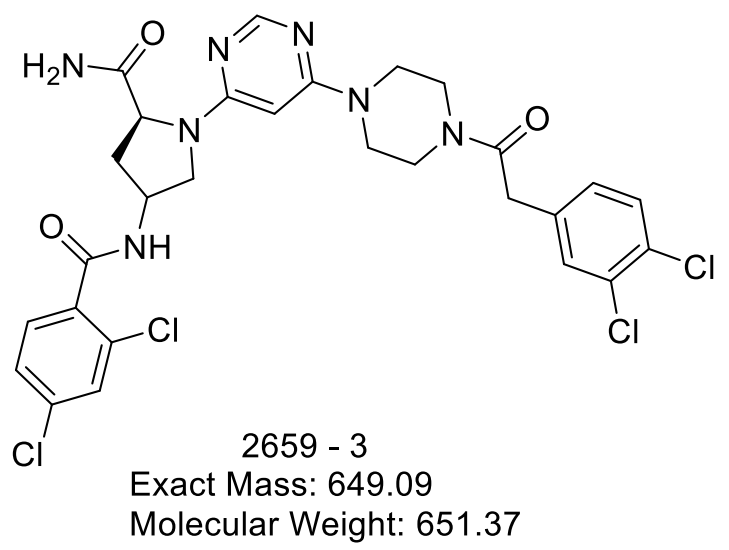

uAU

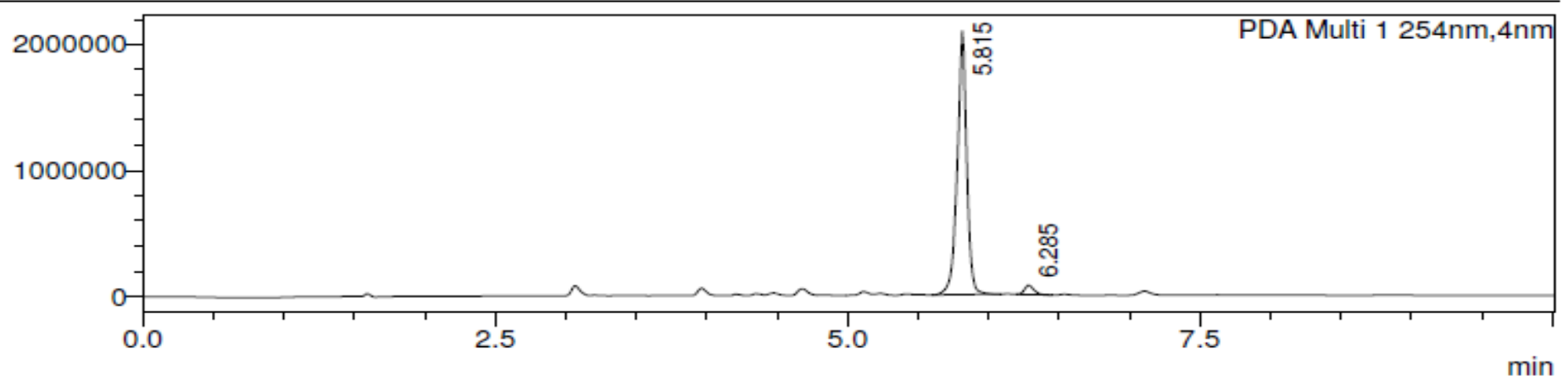

Peak\#: 3 R.Time:5.850(Scan\#: 1405)

MassPeaks: 31

Spectrum Mode:Averaged 5.846-5.854(1404-1406)

BG Mode:Calc Segment 1 - Event 1

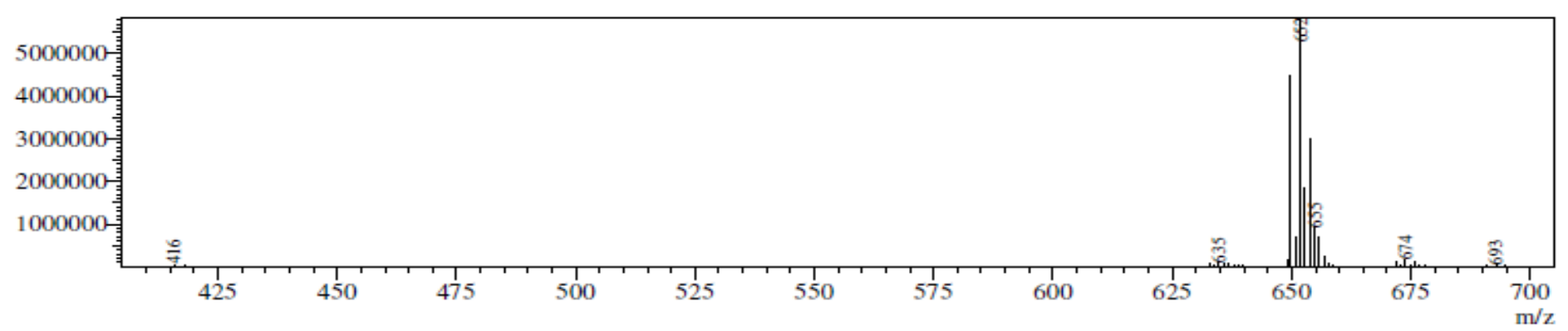


2659-4<smiles>CCC(C)CC(=O)NC1C[C@H](C(N)=O)N(c2cc(N3CCN(C(=O)C(CC)CC)CC3)ncn2)C1</smiles>

Exact Mass: 487.33

Molecular Weight: 487.64

uAU

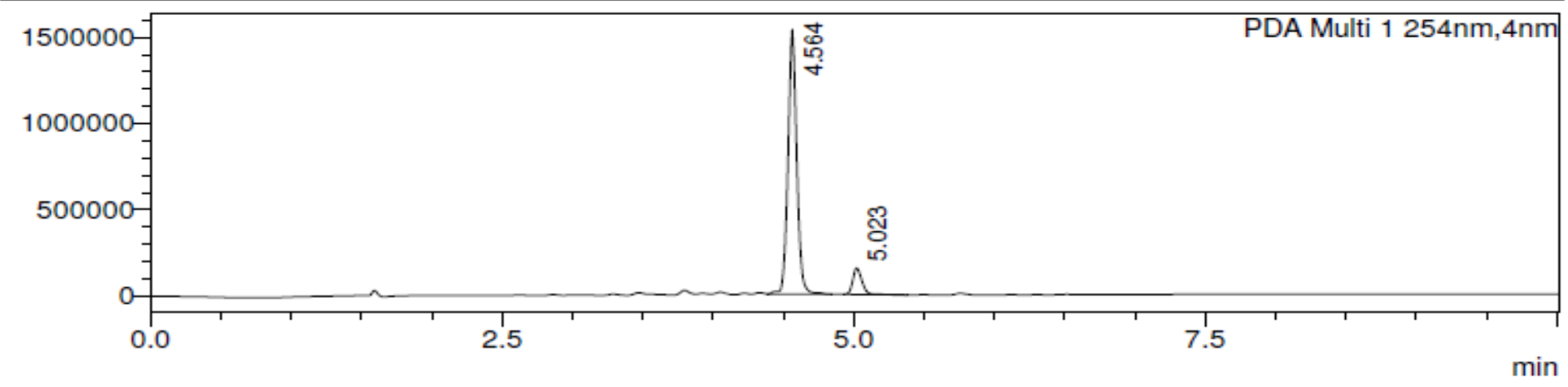

Peak\#:4 R. Time:4.603(Scan\#: 1106)

MassPeaks:33

Spectrum Mode:Averaged 4.600-4.608(1105-1107)

BG Mode:Calc Segment 1 - Event 1

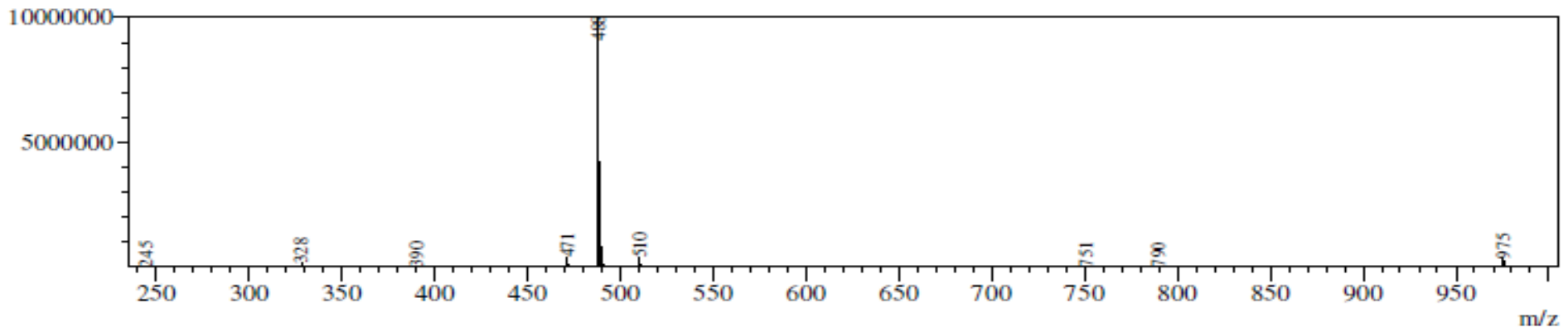


2659-5

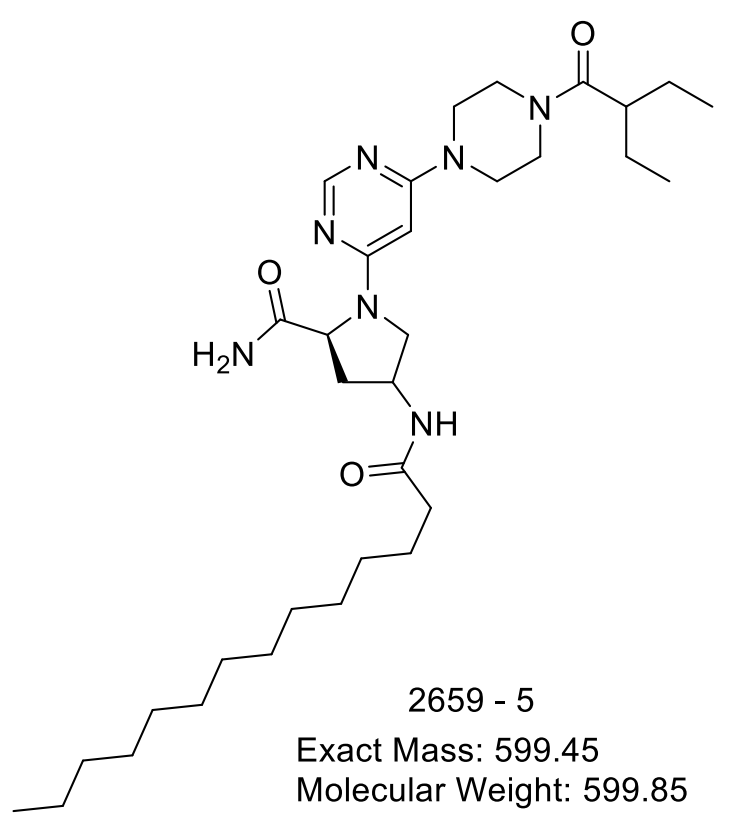

uAU

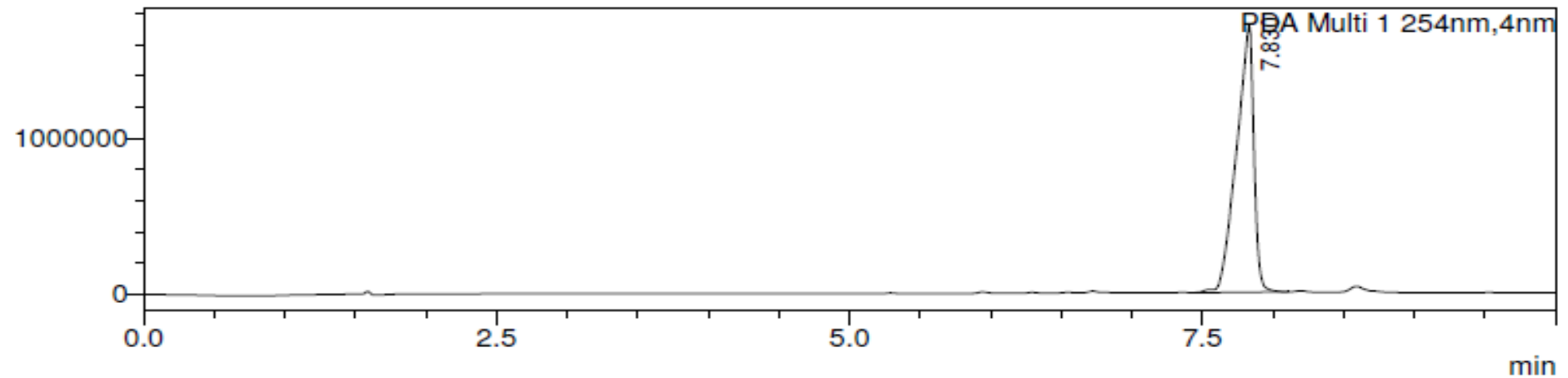

Peak\#:4 R.Time:7.862(Scan\#: 1888)

MassPeaks: 23

Spectrum Mode:Averaged 7.858-7.867(1887-1889)

BG Mode:Calc Segment 1 - Event 1

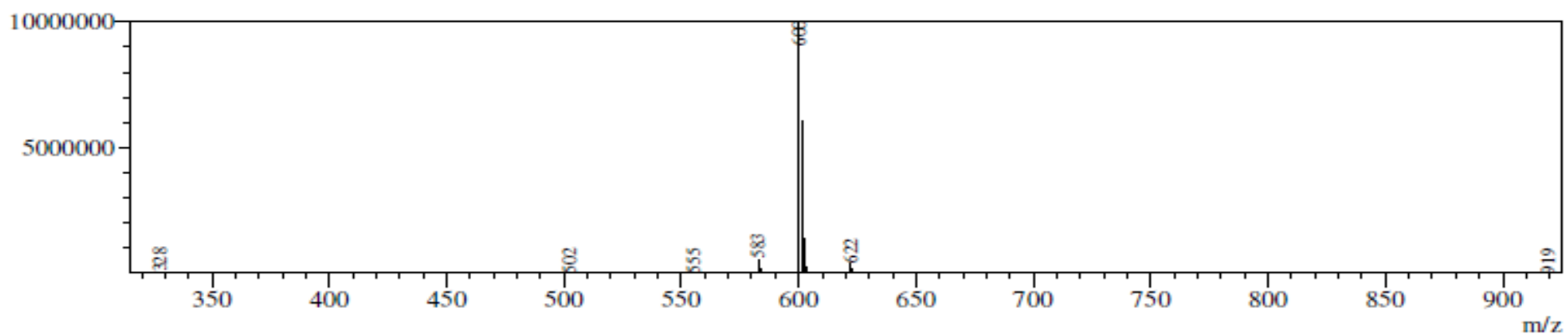




\section{9-6}

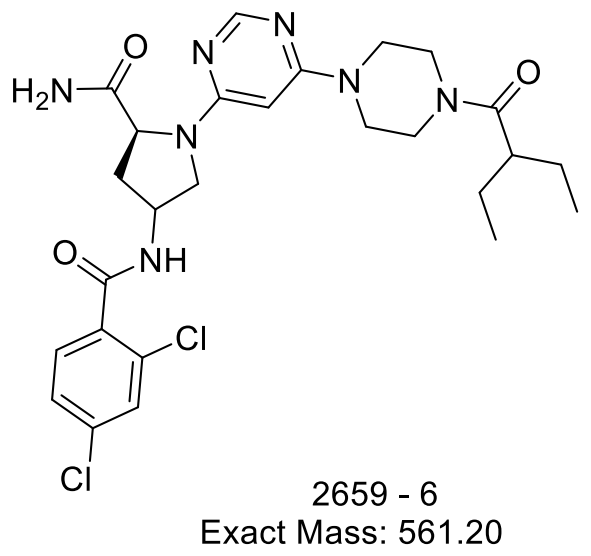

Molecular Weight: 562.49

uAU

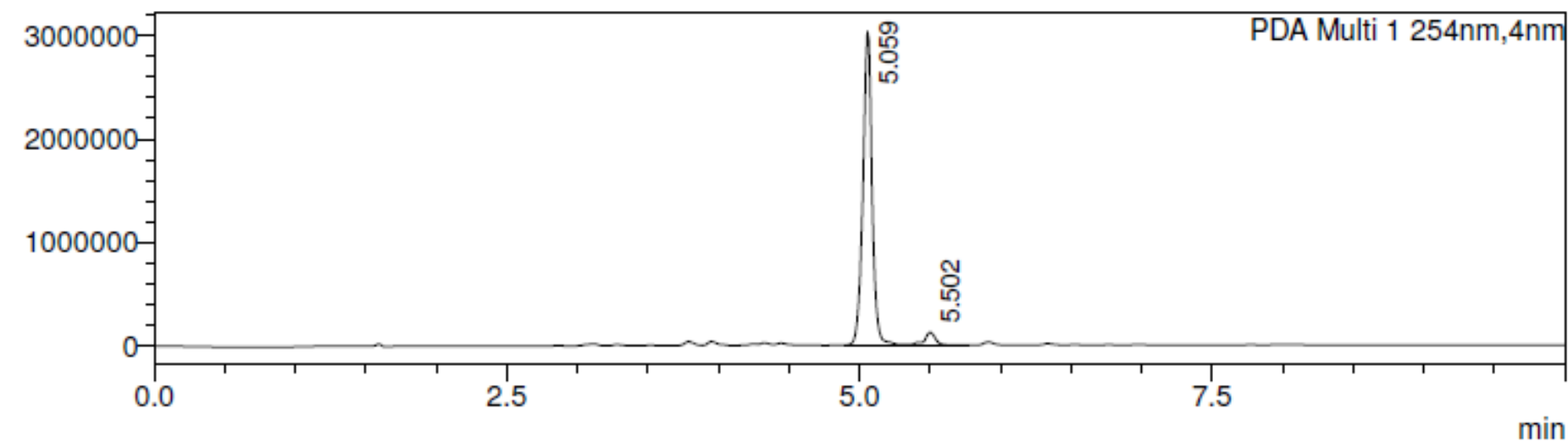

Peak\#:3 R.Time:5.104(Scan\#: 1226)

MassPeaks: 26

Spectrum Mode:Averaged 5.100-5.108(1225-1227)

BG Mode:Calc Segment 1 - Event 1

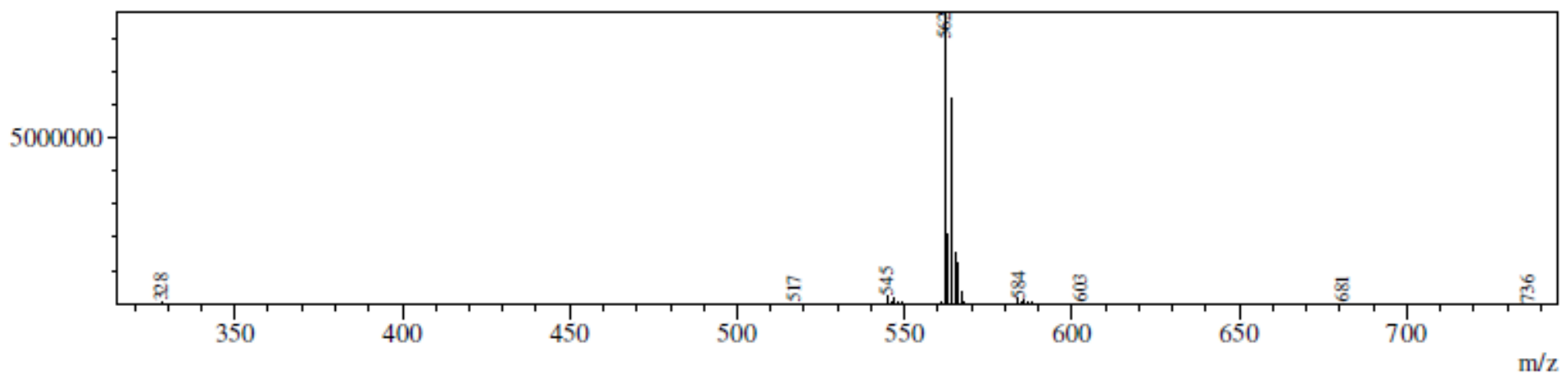




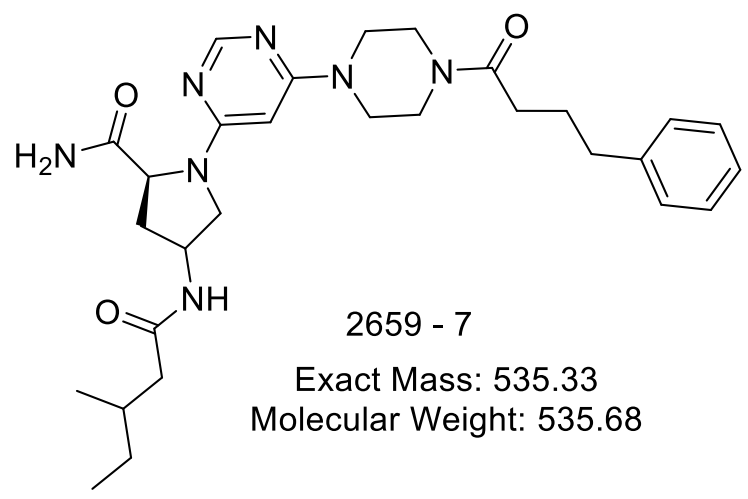

UAU

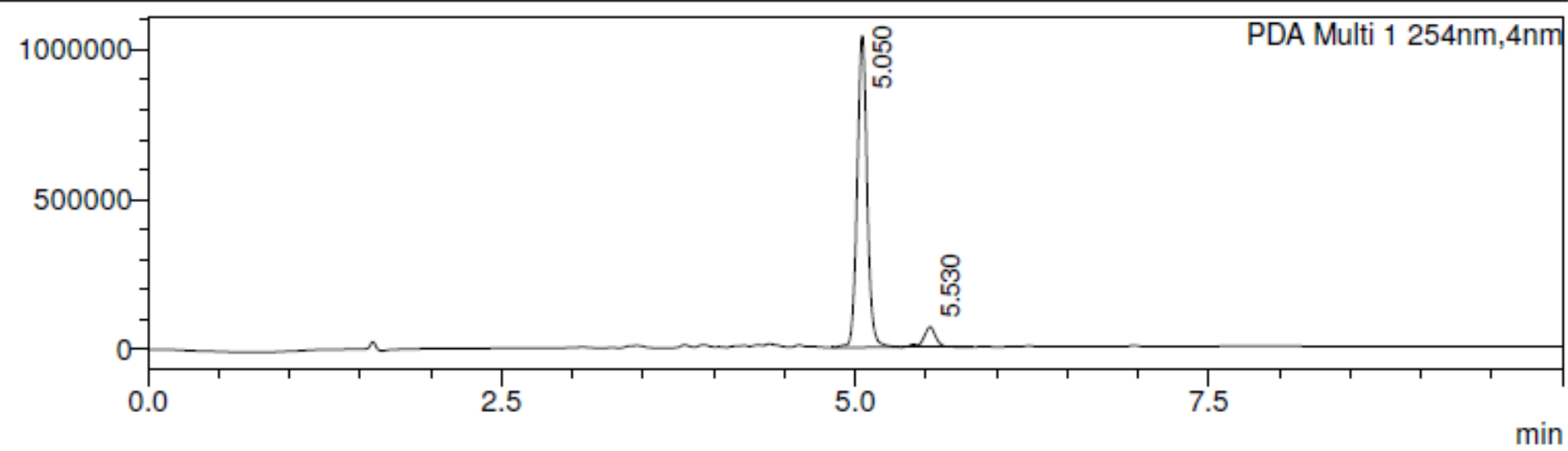

Peak\#:3 R.Time:5.094(Scan\#: 1224)

MassPeaks:31

Spectrum Mode:Averaged 5.092-5.100(1223-1225)

BG Mode:Calc Segment 1 - Event 1

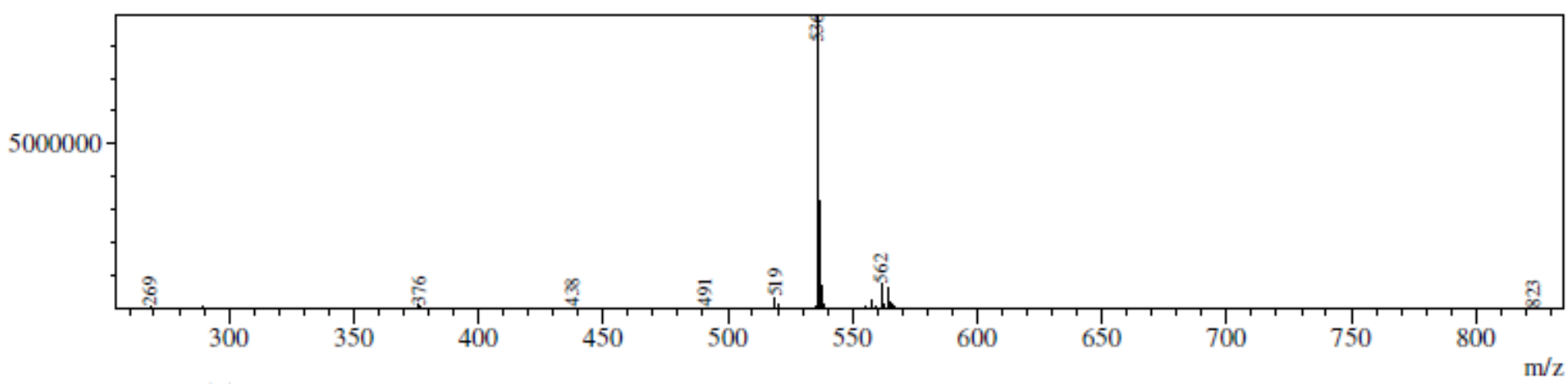


2659-8

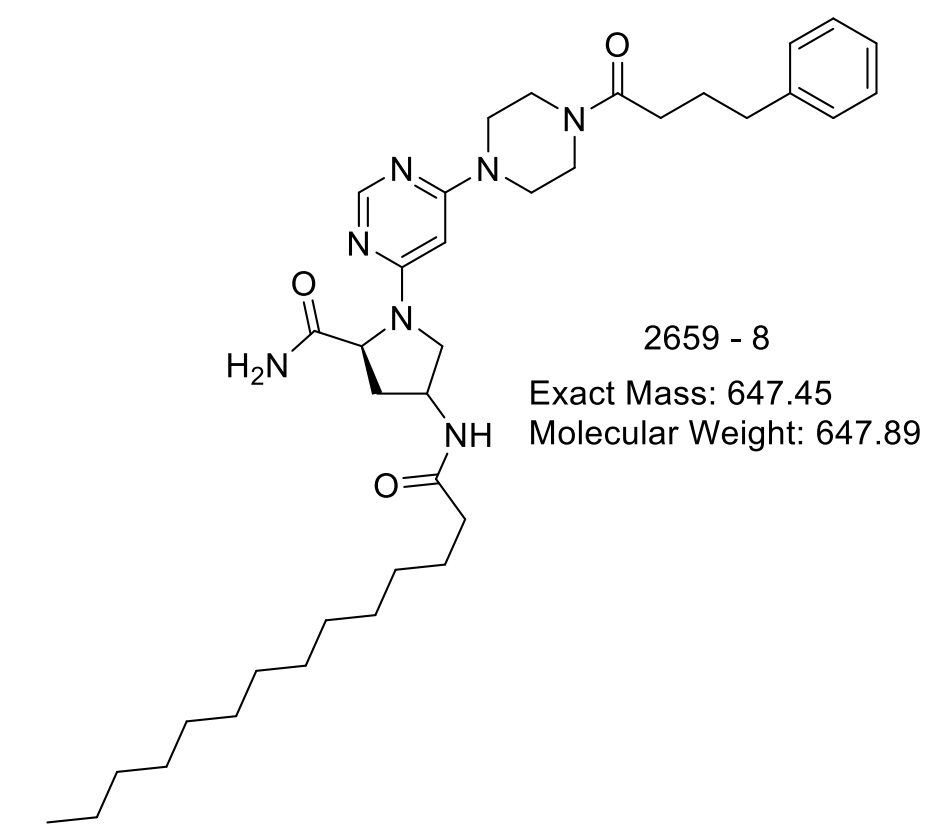

uAU

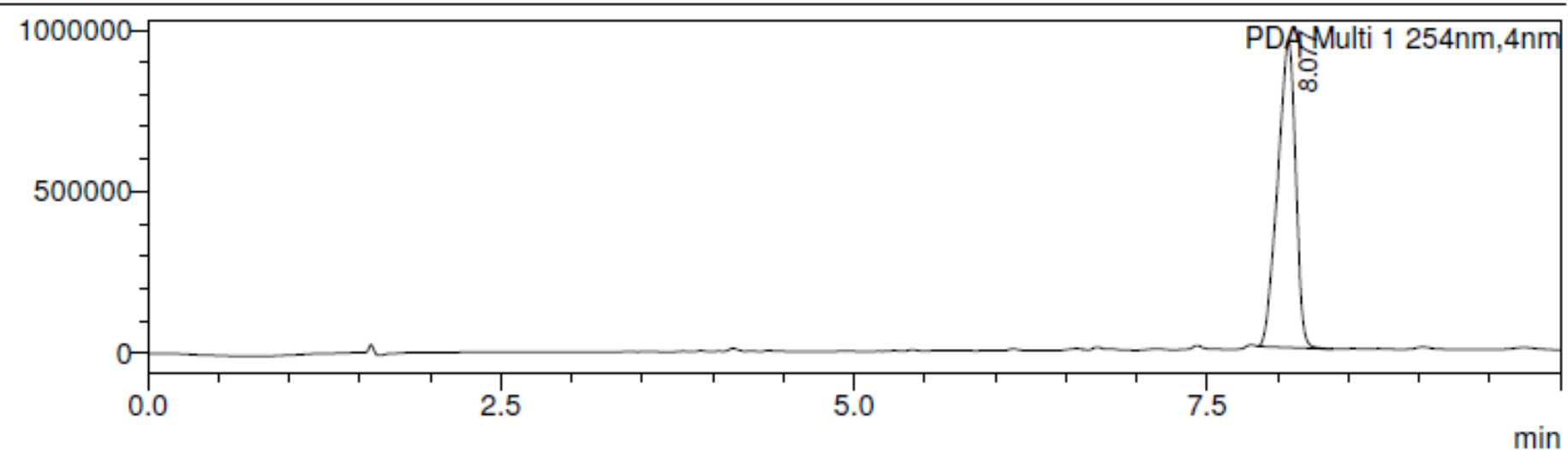

Peak\#:4 R.Time:8.119(Scan\#: 1950)

MassPeaks:21

Spectrum Mode:Averaged 8.117-8.125(1949-1951)

BG Mode:Calc Segment 1 - Event 1

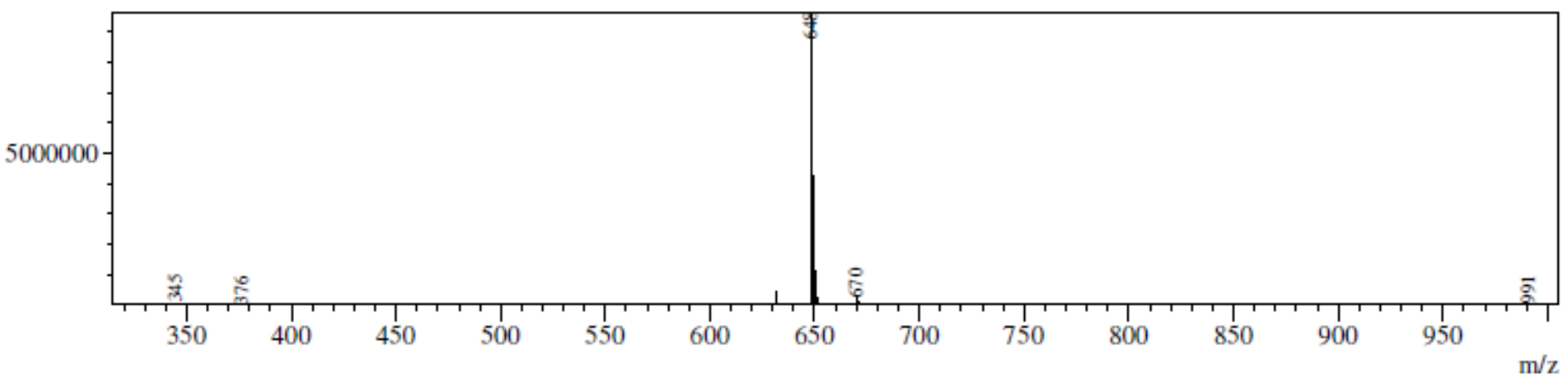




\section{9-9}

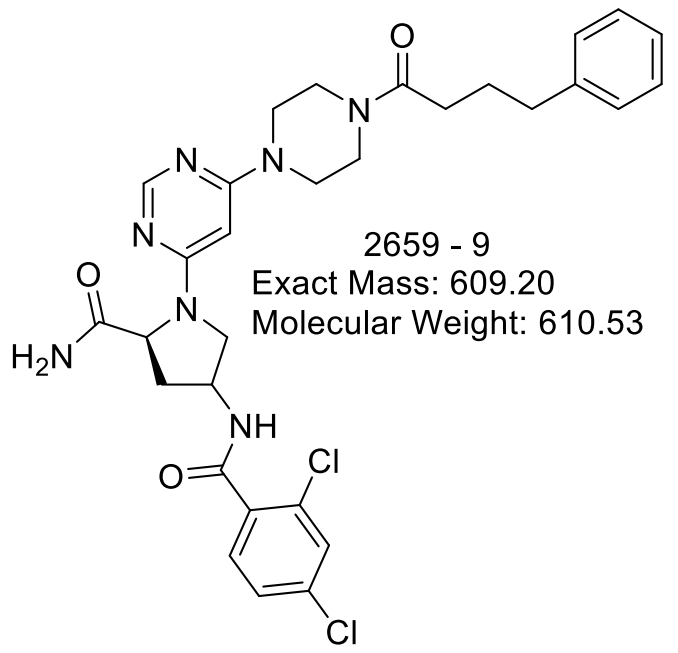

UAU

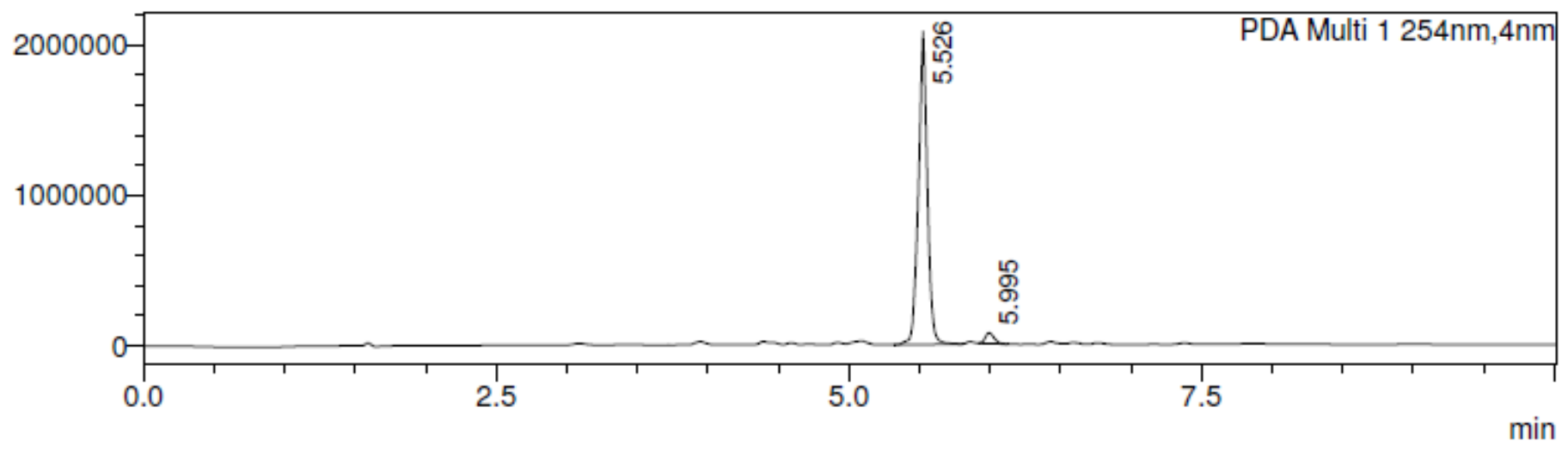

Peak\#:3 R.Time:5.567(Scan\#: 1337)

MassPeaks: 20

Spectrum Mode:Averaged 5.563-5.571(1336-1338)

BG Mode:Calc Segment 1 - Event 1

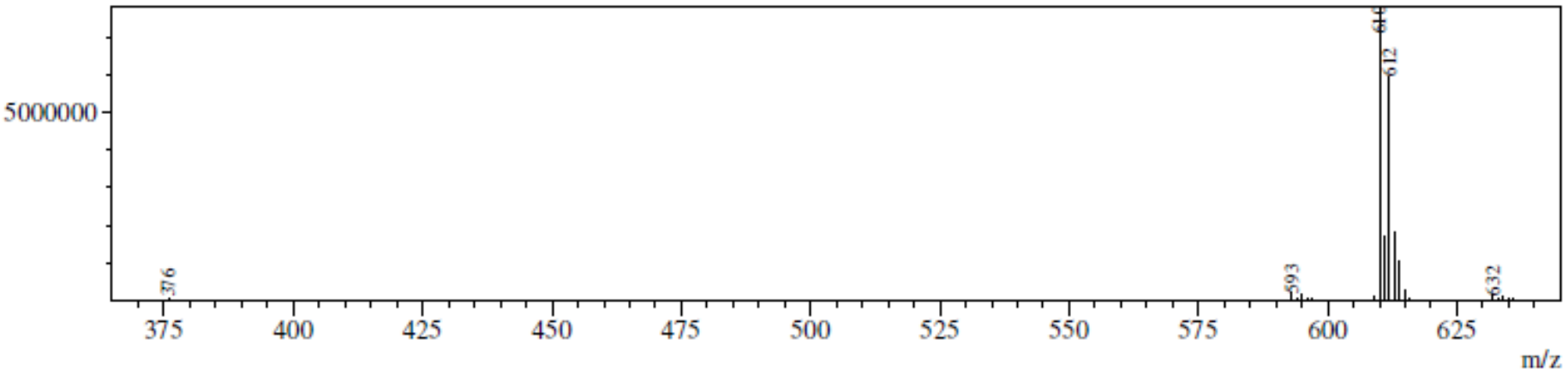




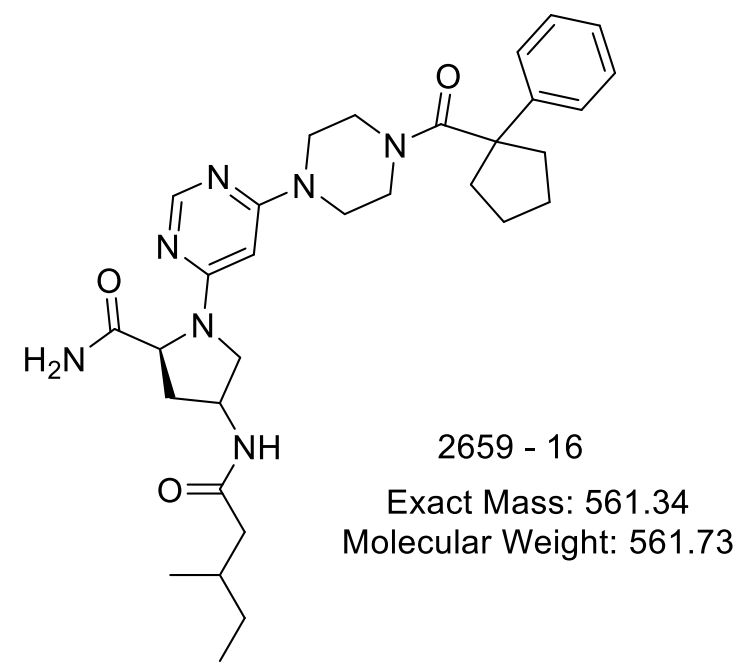

uAU

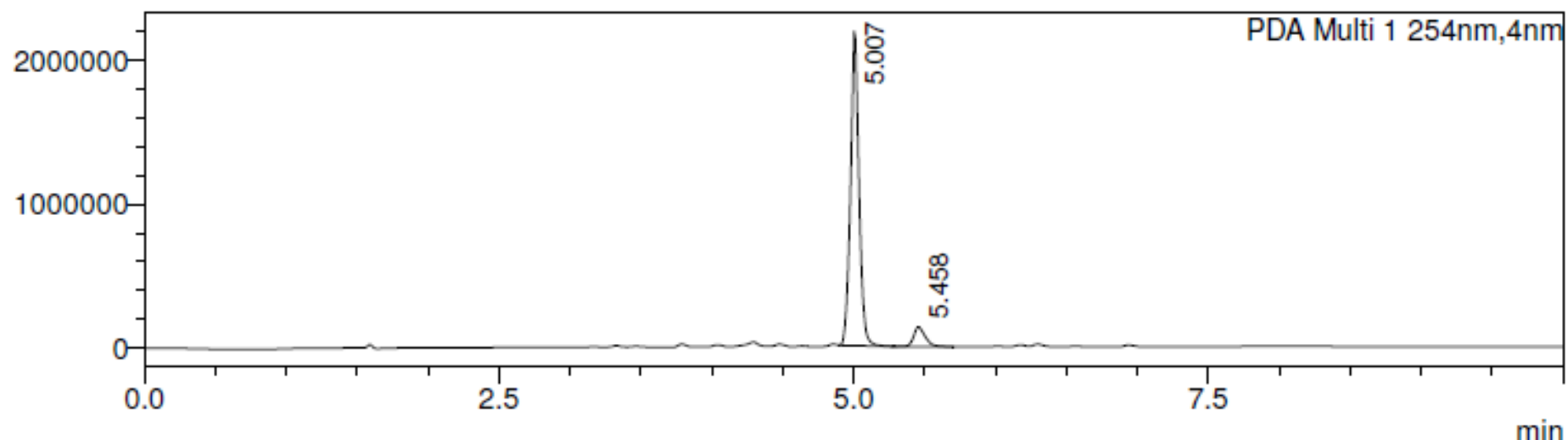

Peak\#:3 R. Time:5.046(Scan\#: 1212)

MassPeaks: 24

Spectrum Mode:Averaged 5.042-5.050(1211-1213)

BG Mode:Calc Segment 1 - Event 1

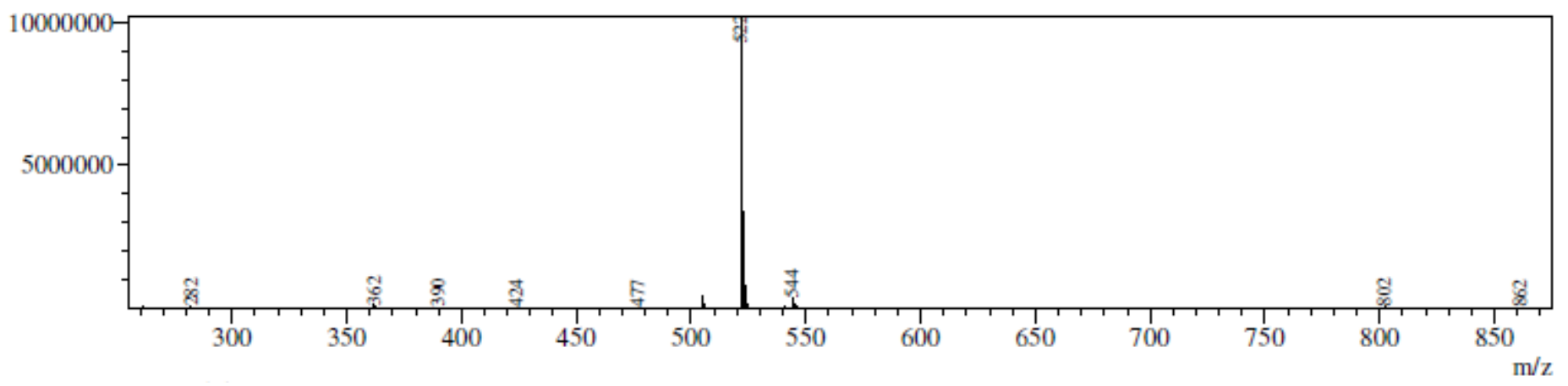




\section{9-11}

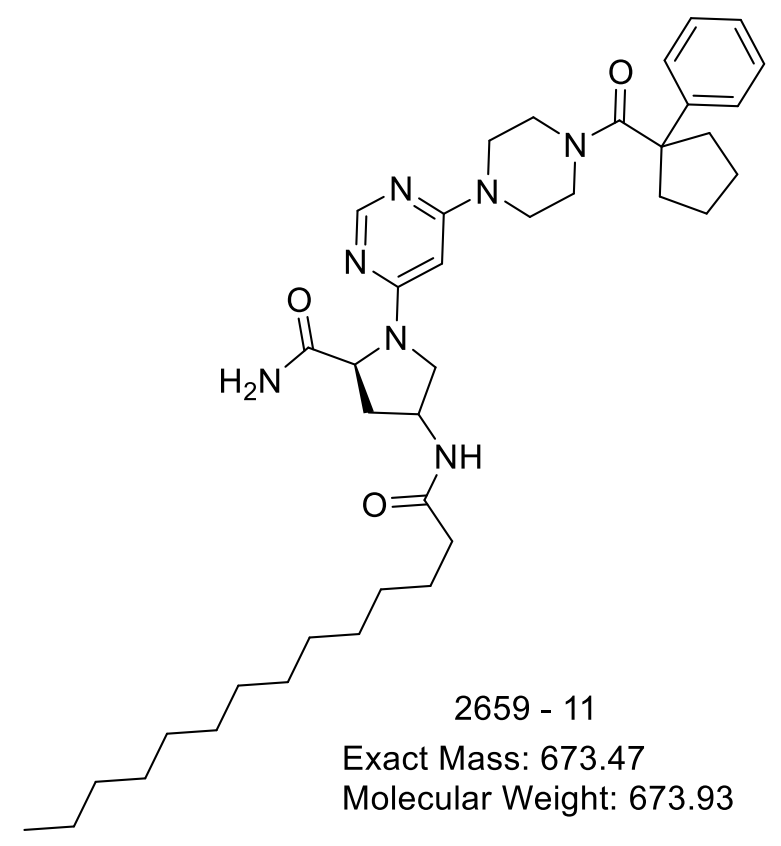

uAU

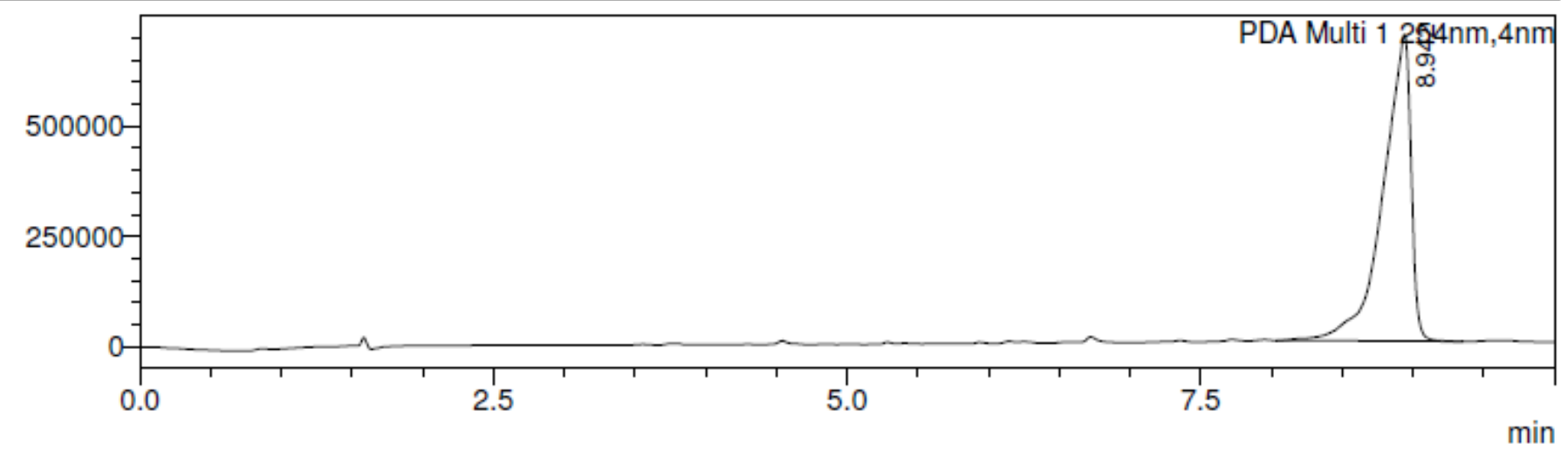

Peak\#:5 R.Time:8.951(Scan\#:2149)

MassPeaks: 23

Spectrum Mode:Averaged 8.946-8.954(2148-2150)

BG Mode:Calc Segment 1 - Event 1

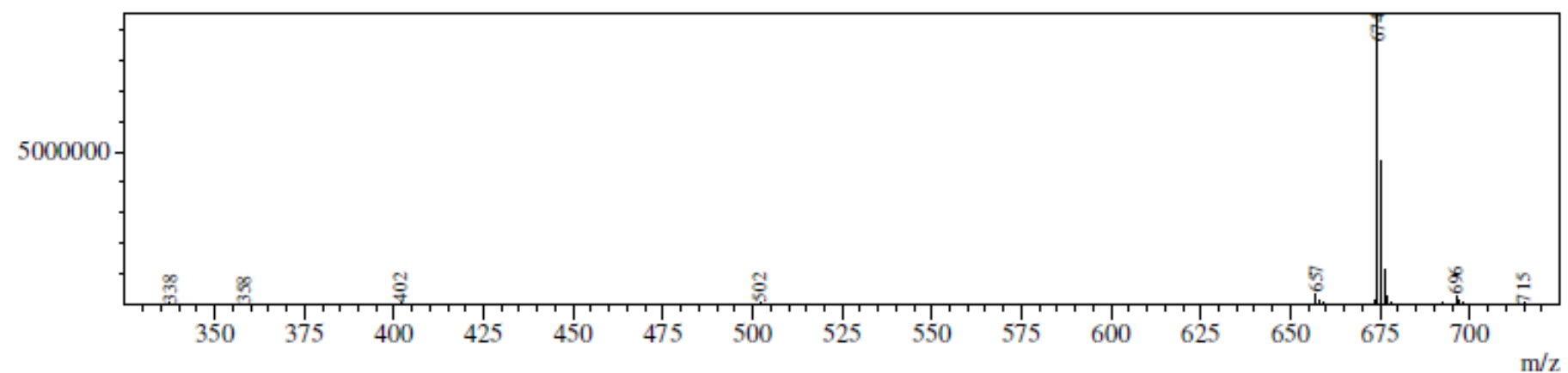


2659-12

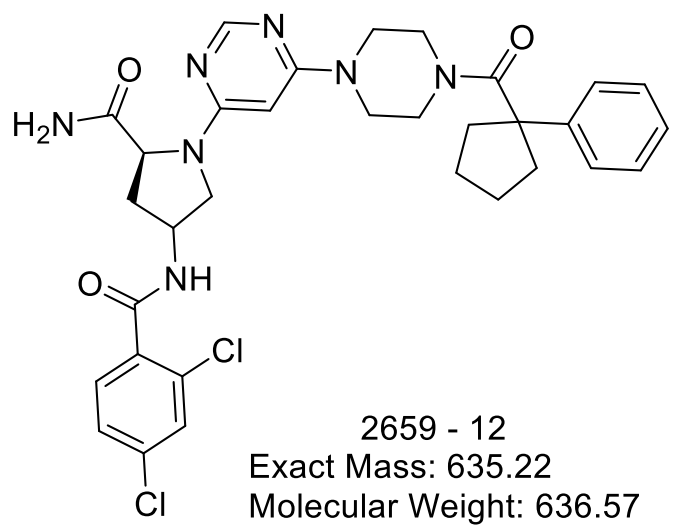

uAU

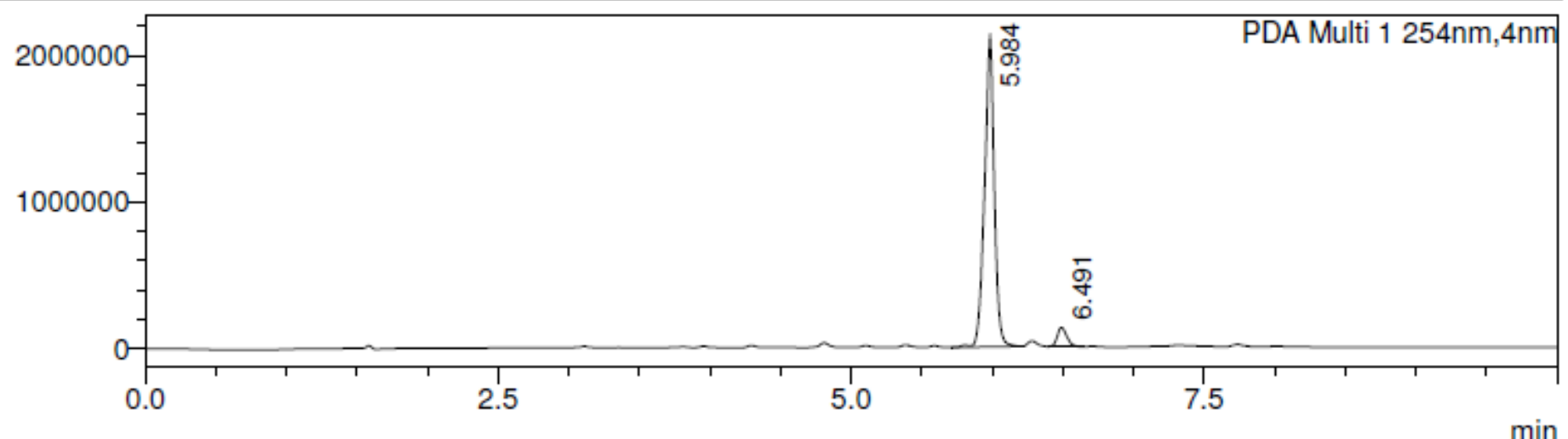

Peak\#: 2 R.Time:6.032(Scan\#: 1449)

MassPeaks: 28

Spectrum Mode:Averaged 6.029-6.038(1448-1450)

BG Mode:Calc Segment 1 - Event 1

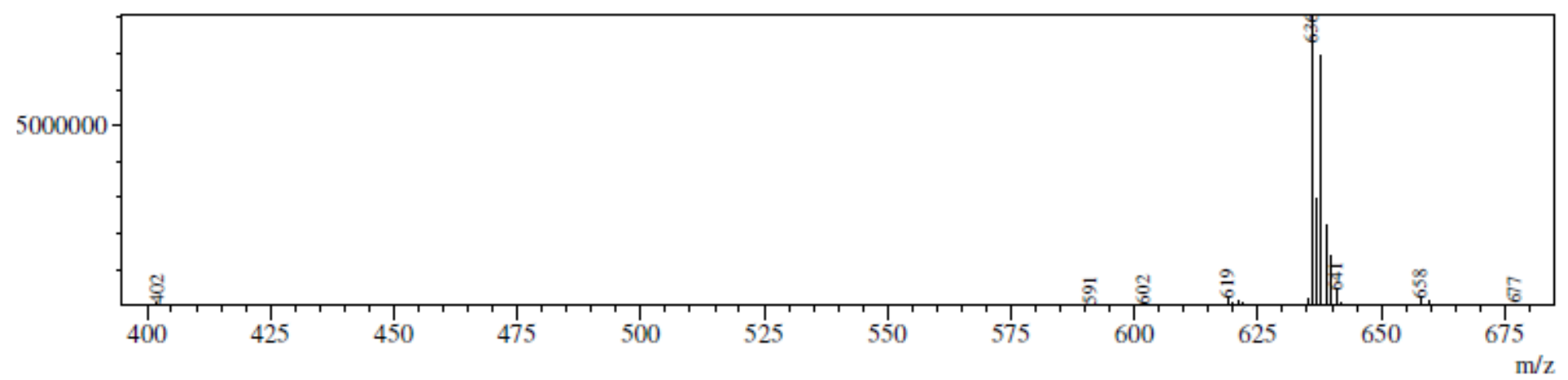




\section{9-13}<smiles>CCC(C)CC(=O)NC1CC(C(N)=O)N(c2cc(N3CCN(C(=O)c4ccc(Cl)cc4Cl)CC3)ncn2)C1</smiles>

uAU

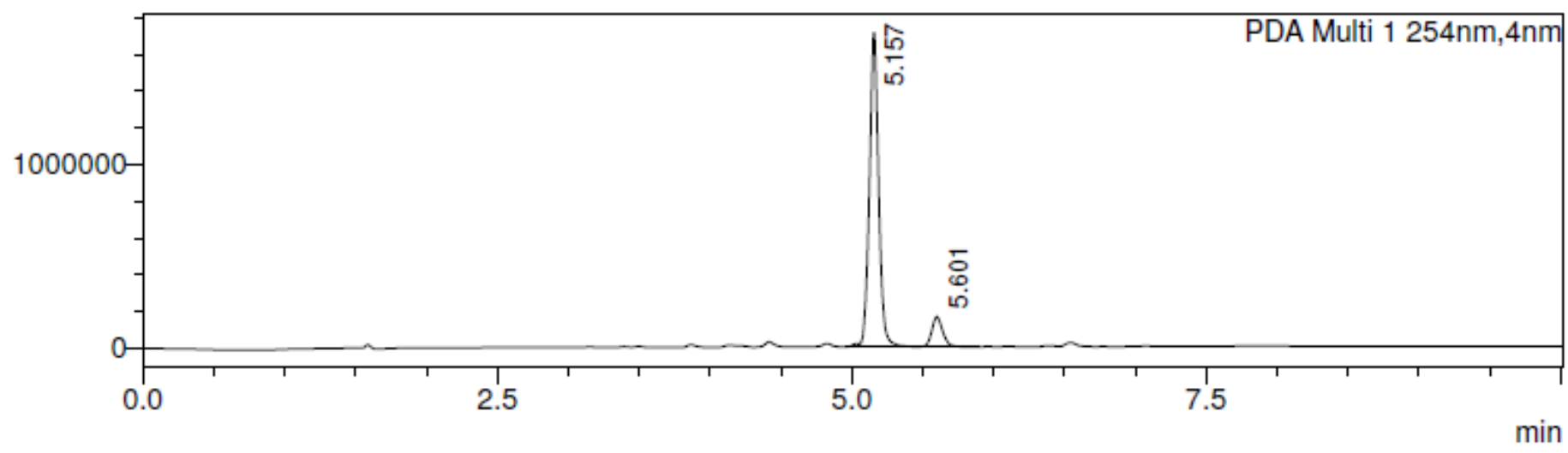

Peak\#:4 R.Time:5.200(Scan\#:1249)

MassPeaks: 27

Spectrum Mode:Averaged 5.196-5.204(1248-1250)

BG Mode:Calc Segment 1 - Event 1

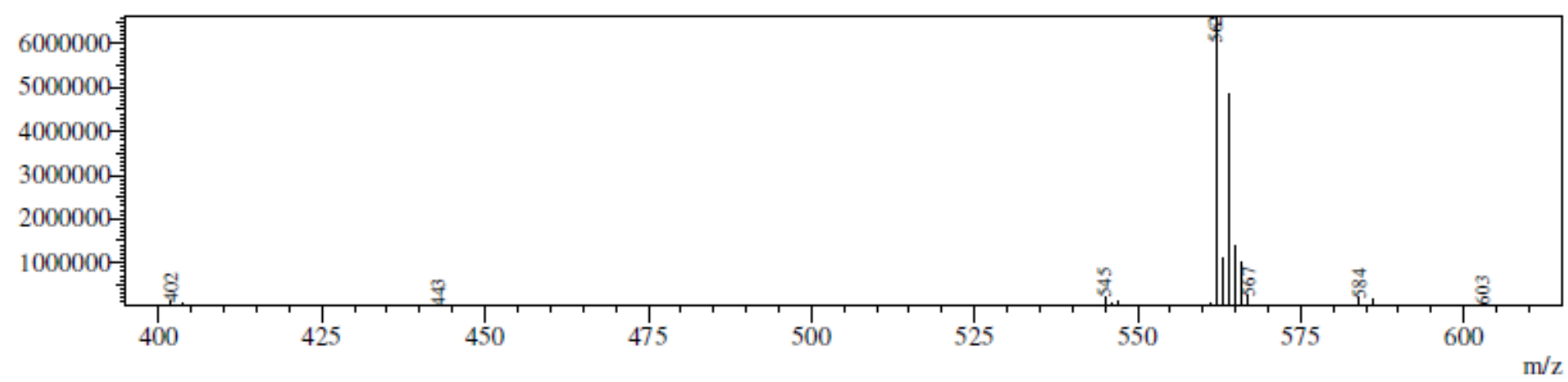


2659-14

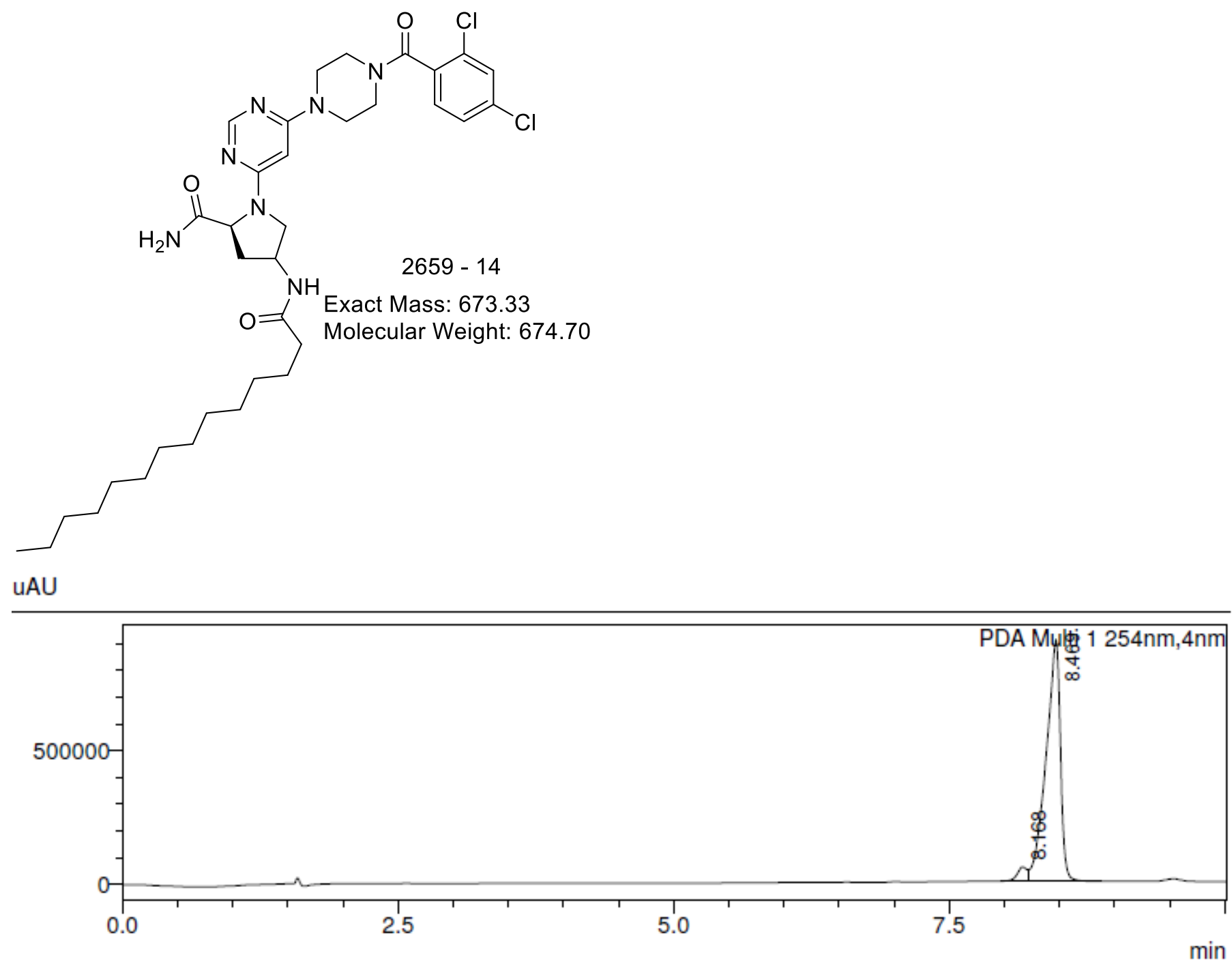

Peak\#:3 R.Time:8.521(Scan\#: 2046)

MassPeaks:27

Spectrum Mode:Averaged 8.517-8.525(2045-2047)

BG Mode:Calc Segment 1 - Event 1

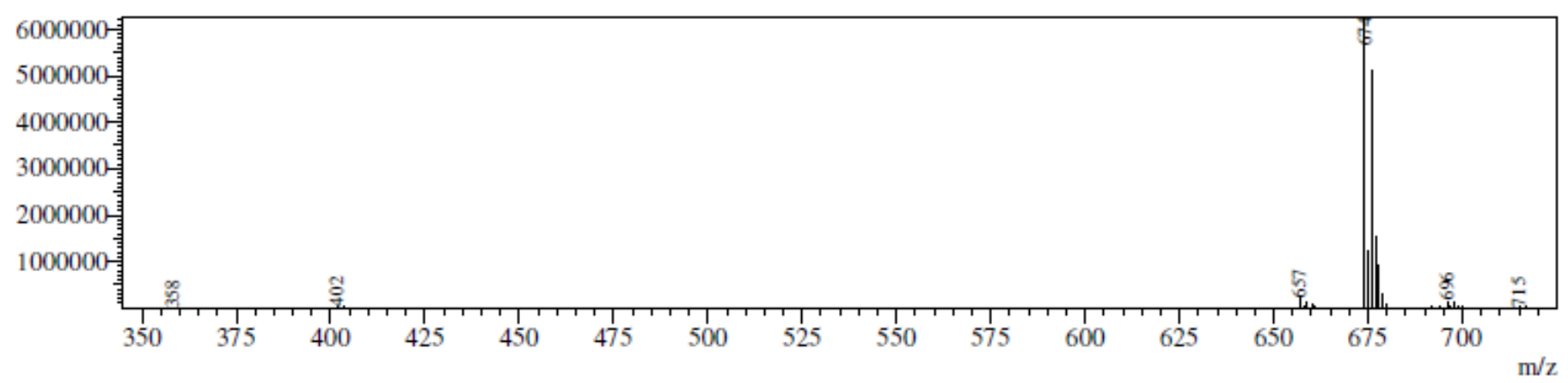




\section{9-15}

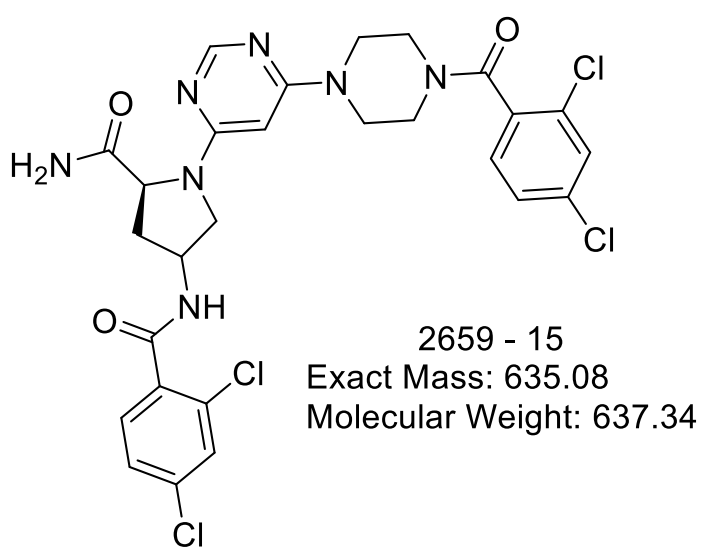

uAU

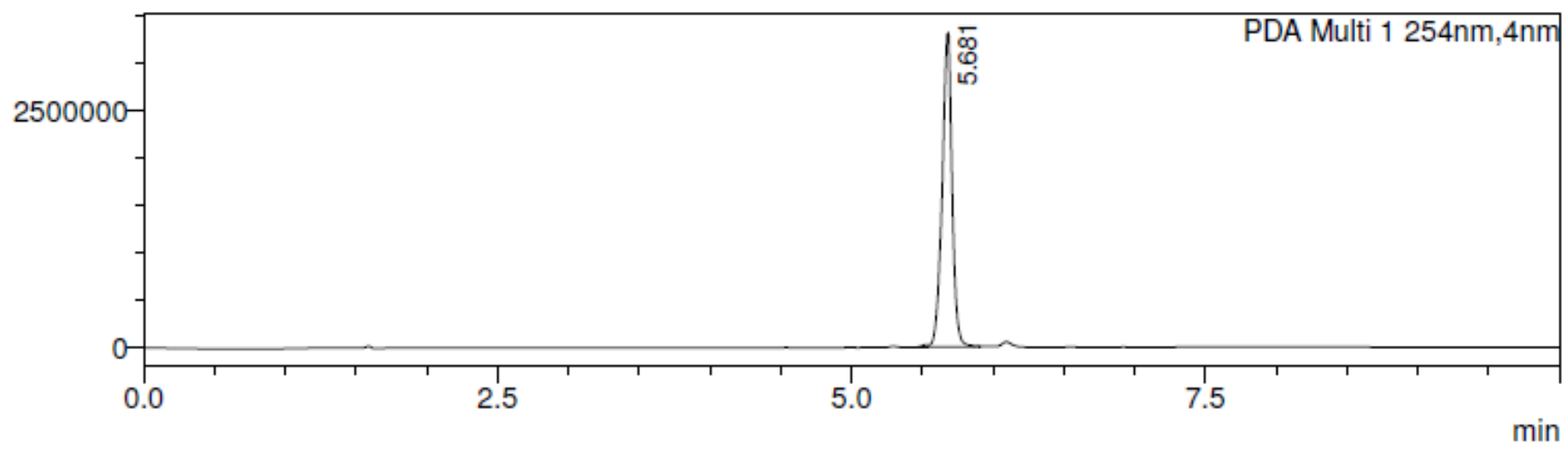

Peak\#:3 R.Time:5.718(Scan\#: 1373)

MassPeaks: 32

Spectrum Mode:Averaged 5.713-5.721(1372-1374)

BG Mode:Calc Segment 1 - Event 1

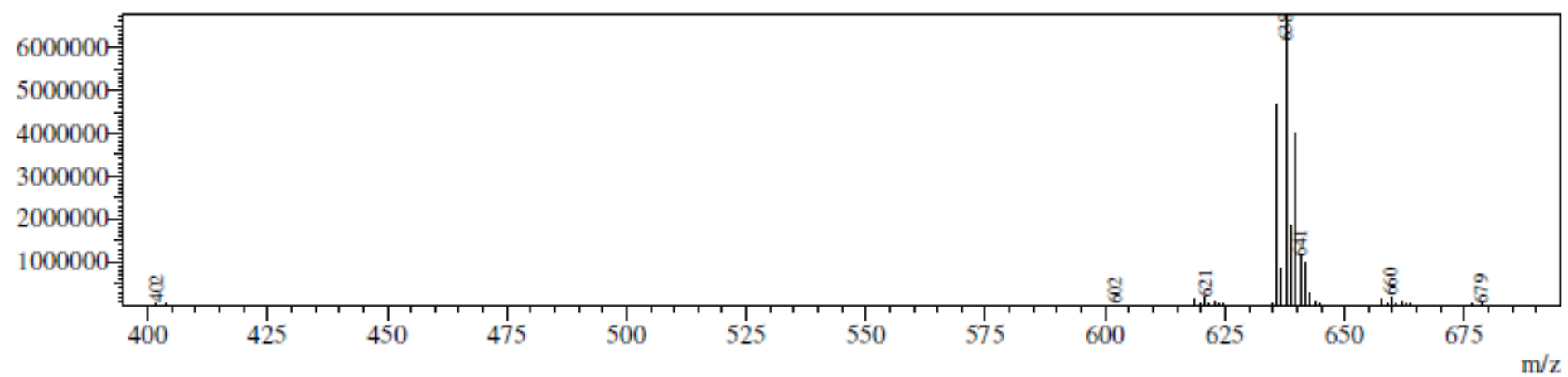


2659-16

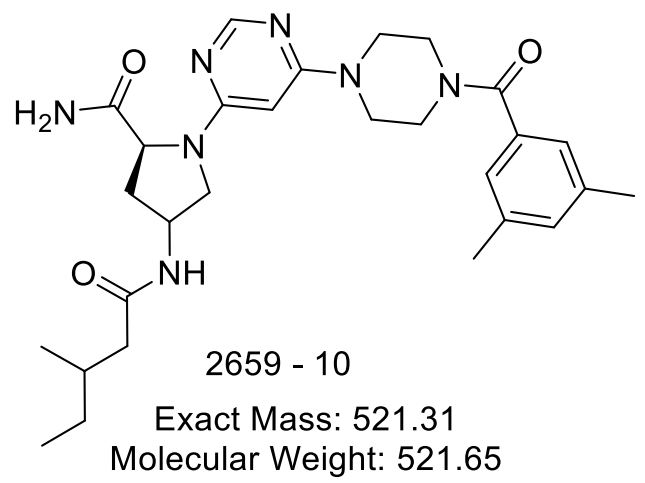

uAU

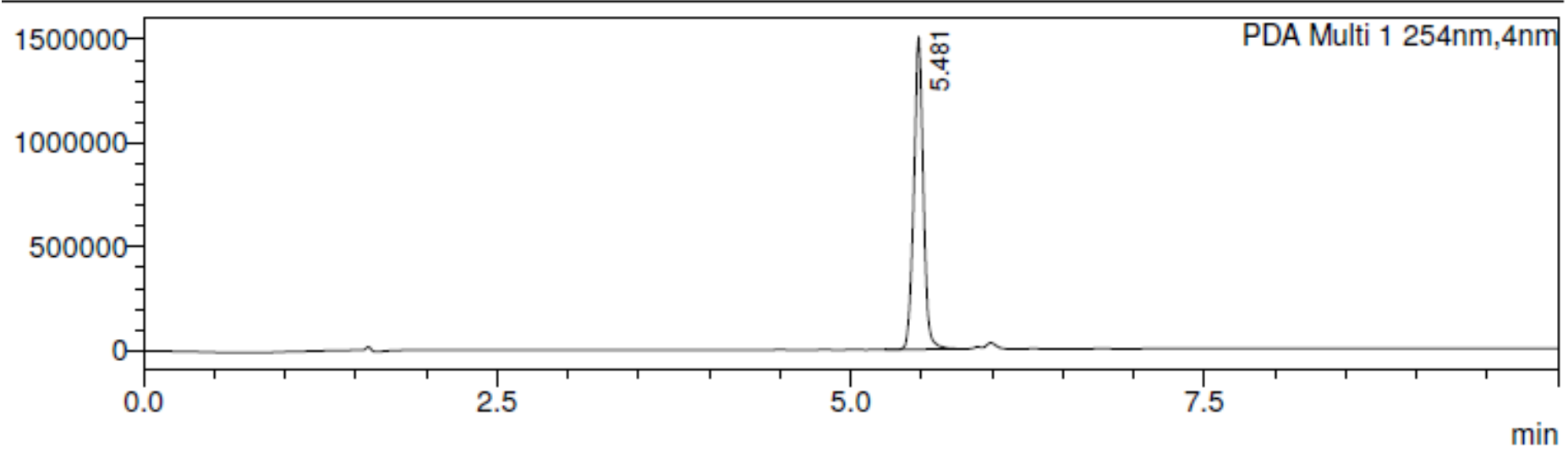

Peak\#: $1 \quad$ R.Time:5.528(Scan\#: 1328)

MassPeaks: 25

Spectrum Mode:Averaged 5.525-5.533(1327-1329)

BG Mode:Calc Segment 1 - Event 1

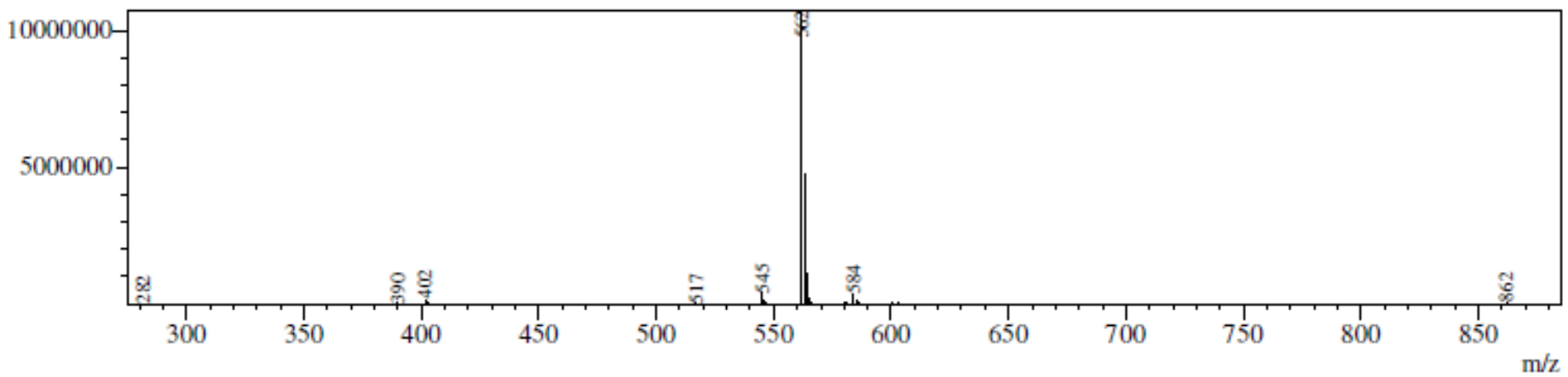




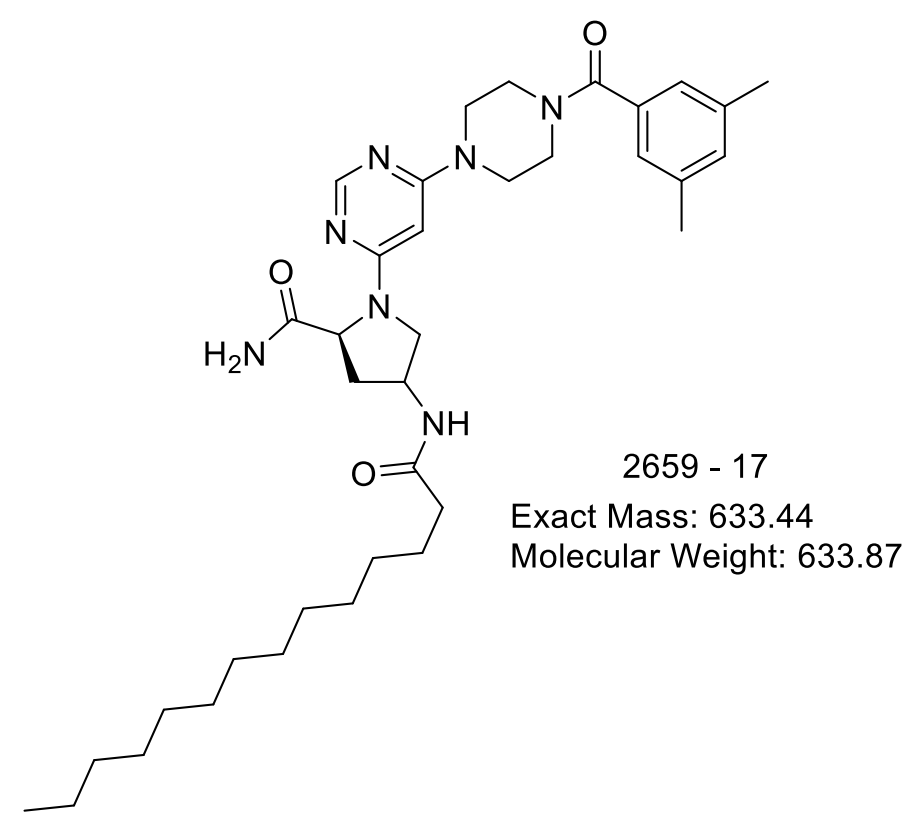

uAU

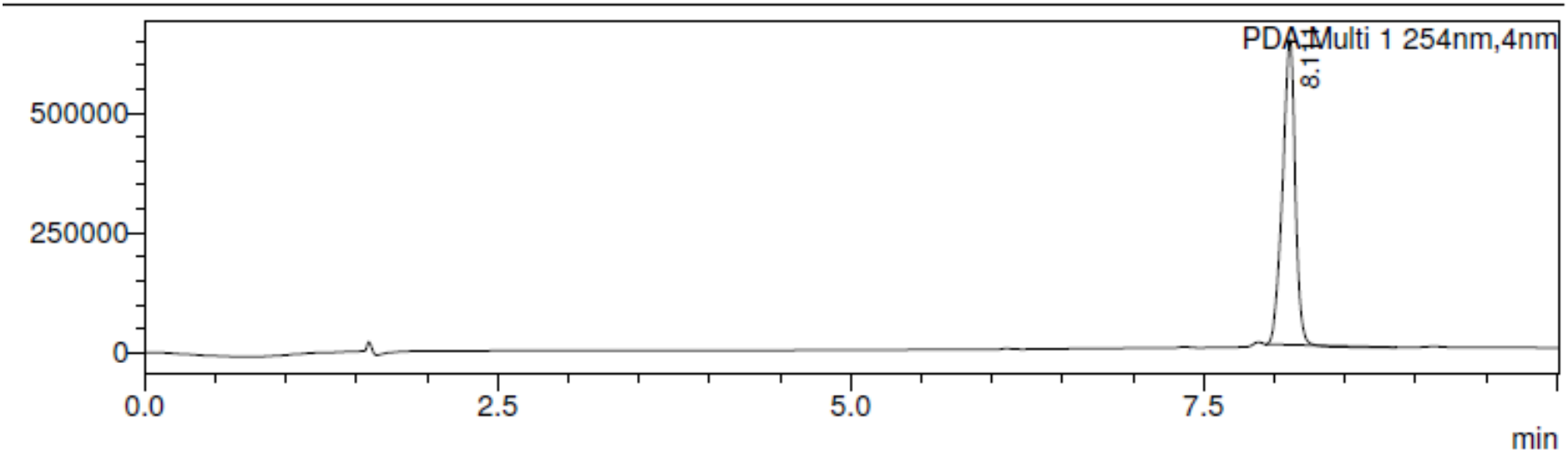

Peak\#:5 R.Time:8.152(Scan\#: 1957)

MassPeaks: 22

Spectrum Mode:Averaged 8.146-8.154(1956-1958)

BG Mode:Calc Segment 1 - Event 1

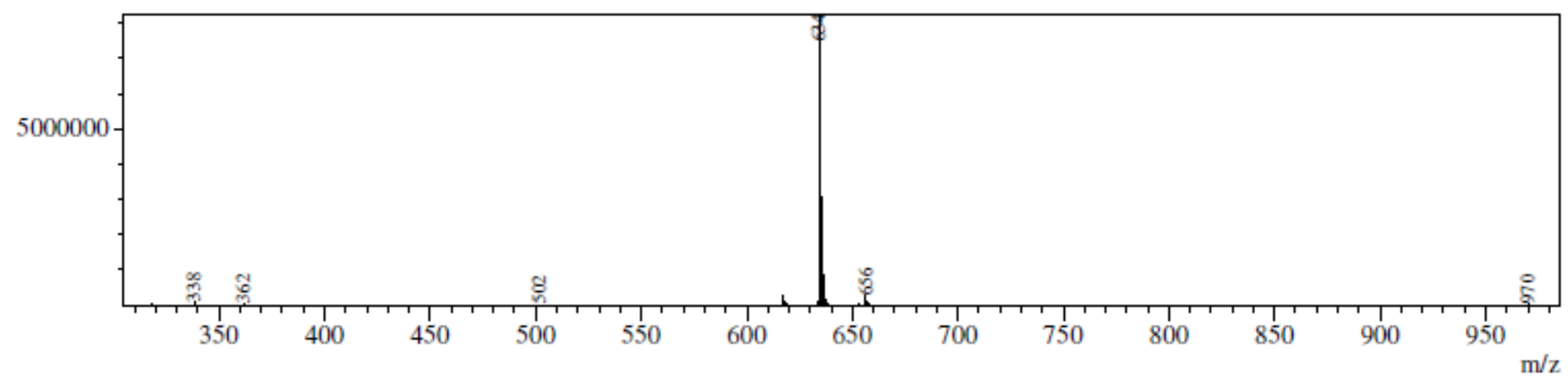


2659-18

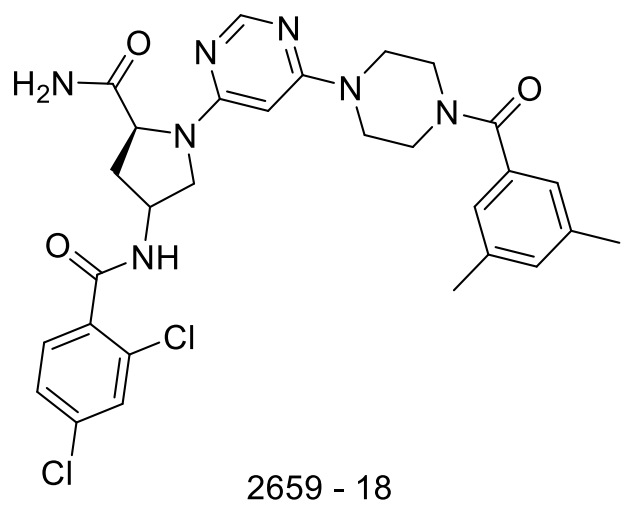

Exact Mass: 595.19

Molecular Weight: 596.51

uAU

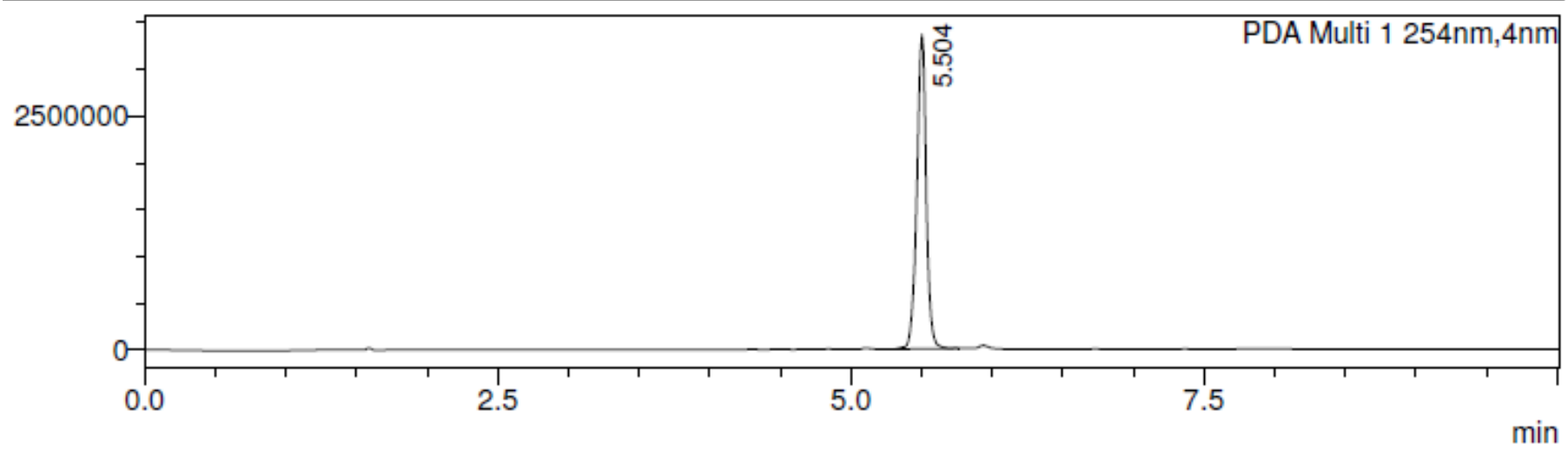

Peak\#:4 R.Time:5.542(Scan\#: 1331)

MassPeaks: 30

Spectrum Mode:Averaged 5.538-5.546(1330-1332)

BG Mode:Calc Segment 1 - Event 1

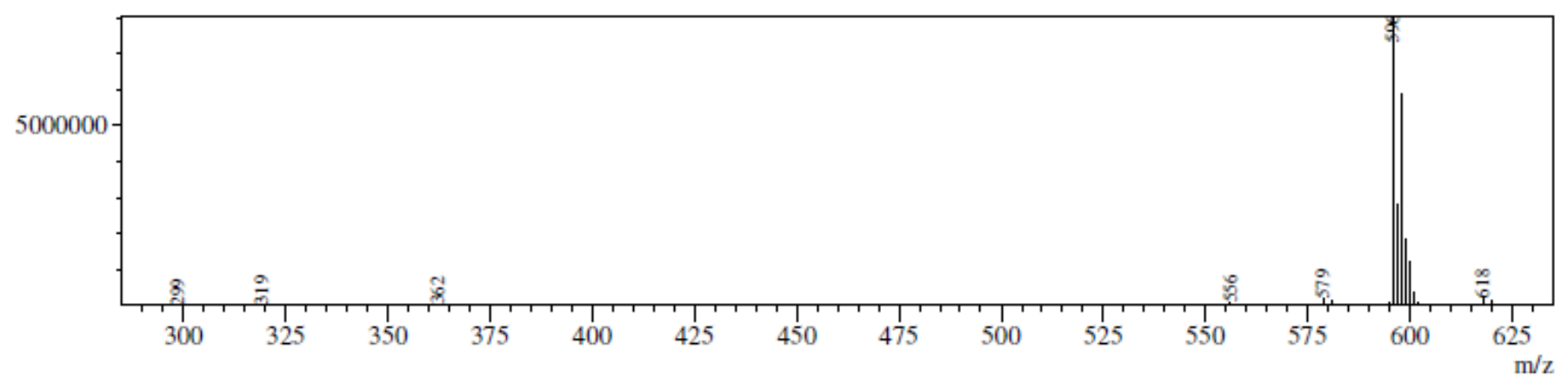


LCMS of lead compound aliquot (after 16 months from the date of synthesis)
Date Acquired

Sample Name

Sample ID

Injection Volume

Data File

Method File

sample inrormation

17 pure

2659-pure 17.lcd

6 min 5-95,1Min Clean(254)_(214) COL1.Icm (l) $\mathrm{N}$<smiles>NC(=O)C1CCCN1</smiles>

Chromatogran

2659

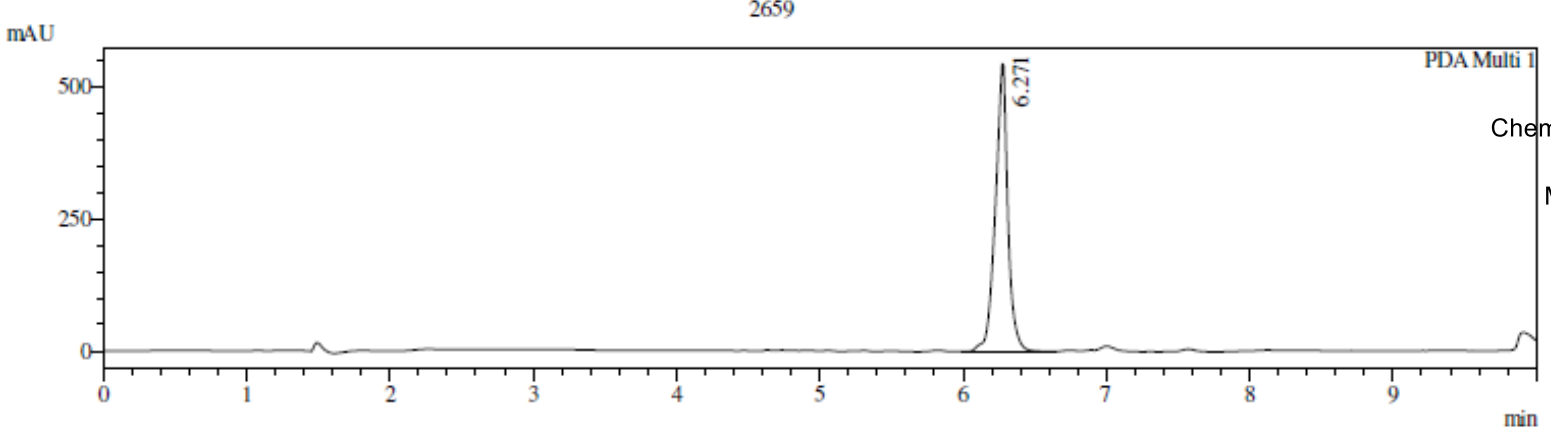

mAU

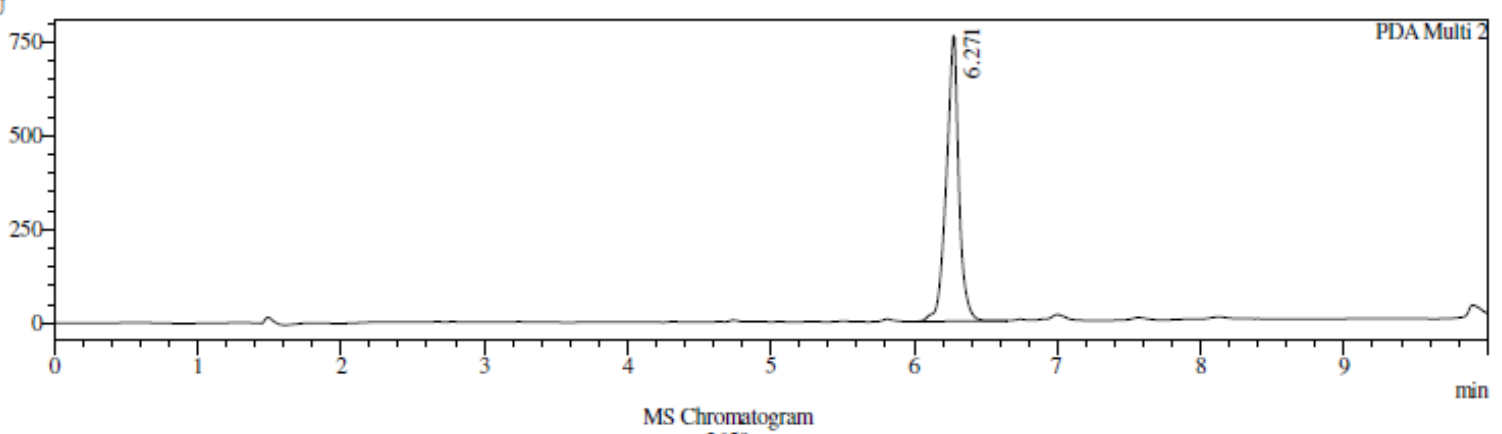

2659

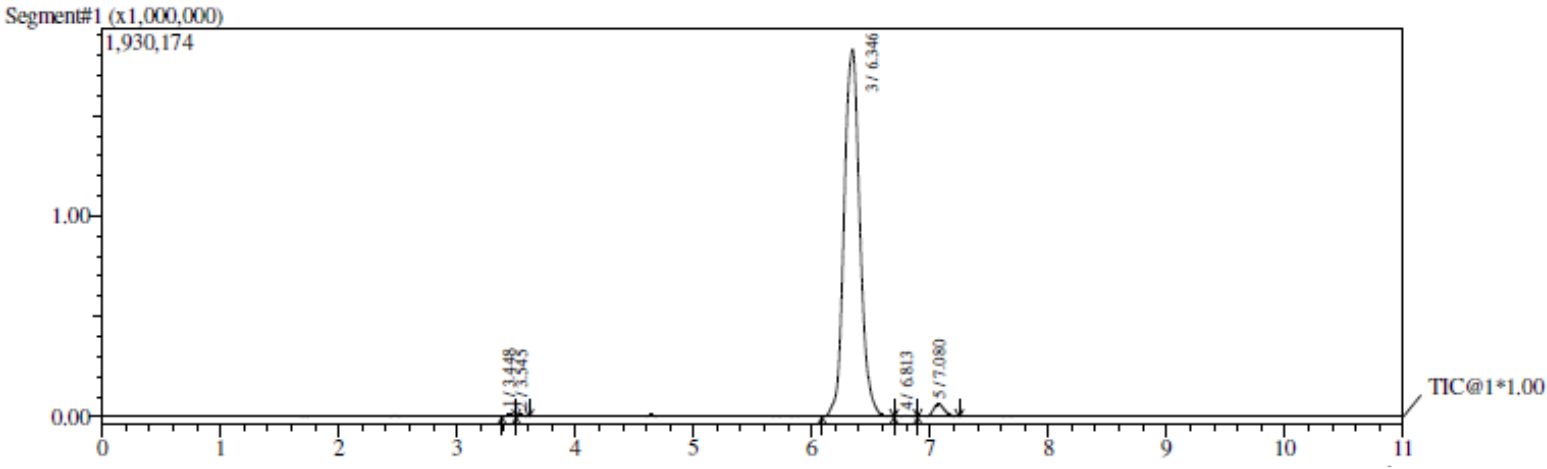

Peak\#:3 Ret.Time:Averaged 6.343-6.350(Scan\#.1904-1906)

BG ModeCalc 6.087 $<->6.697(1827<->2010)$

Mass Peaks:9 Base Peak:634.30(1142820) Polarity:Pos Segment1 - Event1

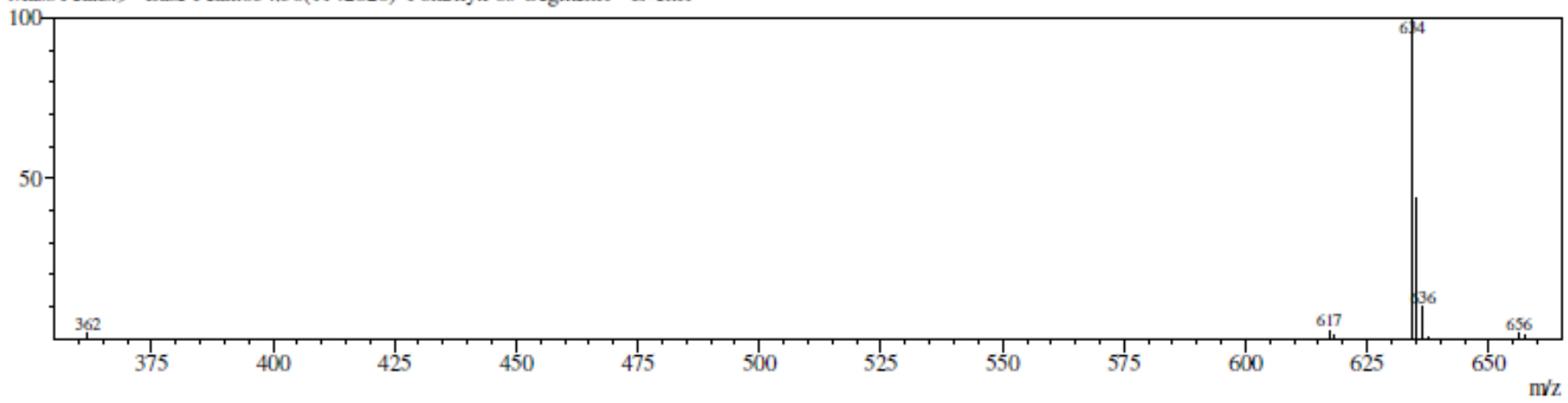

